\title{
Scattering of graphene plasmons at abrupt interfaces: An analytic and numeric study
}

\author{
Chaves, A. J.; Amorim, B.; Bludov, Yu V.; Gonçalves, P. A. D.; Peres, N. M. R.
}

Published in:

Physical Review B

Link to article, DOI:

10.1103/PhysRevB.97.035434

Publication date:

2018

Document Version

Publisher's PDF, also known as Version of record

Link back to DTU Orbit

Citation (APA):

Chaves, A. J., Amorim, B., Bludov, Y. V., Gonçalves, P. A. D., \& Peres, N. M. R. (2018). Scattering of graphene plasmons at abrupt interfaces: An analytic and numeric study. Physical Review B, 97(3), [035434].

https://doi.org/10.1103/PhysRevB.97.035434

\section{General rights}

Copyright and moral rights for the publications made accessible in the public portal are retained by the authors and/or other copyright owners and it is a condition of accessing publications that users recognise and abide by the legal requirements associated with these rights.

- Users may download and print one copy of any publication from the public portal for the purpose of private study or research.

- You may not further distribute the material or use it for any profit-making activity or commercial gain

- You may freely distribute the URL identifying the publication in the public portal 


\title{
Scattering of graphene plasmons at abrupt interfaces: An analytic and numeric study
}

\author{
A. J. Chaves, ${ }^{1, *}$ B. Amorim, ${ }^{2, \dagger}$ Yu. V. Bludov,${ }^{1, \ddagger}$ P. A. D. Gonçalves,${ }^{3,4, \pi l}$ and N. M. R. Peres ${ }^{1, \S}$ \\ ${ }^{1}$ Center and Departament of Physics, and QuantaLab, University of Minho, Campus of Gualtar, 4710-057 Braga, Portugal \\ ${ }^{2}$ CeFEMA, Instituto Superior Técnico, Universidade de Lisboa, Av. Rovisco Pais, 1049-001 Lisboa, Portugal \\ ${ }^{3}$ Department of Photonics Engineering and Center for Nanostructured Graphene, \\ Technical University of Denmark, DK-2800 Kgs. Lyngby, Denmark \\ ${ }^{4}$ Centre for Nano Optics, University of Southern Denmark, Campusvej 55, DK-5230 Odense M, Denmark
}

(Received 28 November 2017; published 23 January 2018)

\begin{abstract}
We discuss the scattering of graphene surface plasmon polaritons (SPPs) at an interface between two semiinfinite graphene sheets with different doping levels and/or different underlying dielectric substrates. We take into account retardation effects and the emission of free radiation in the scattering process. We derive approximate analytic expressions for the reflection and the transmission coefficients of the SPPs as well as the same quantities for the emitted free radiation. We show that the scattering problem can be recast as a Fredholm equation of the second kind. Such an equation can then be solved by a series expansion, with the first term of the series corresponding to our approximated analytical solution for the reflection and transmission amplitudes. We have found that almost no free radiation is emitted in the scattering process and that under typical experimental conditions the back-scattered SPP transports very little energy. This paper provides a theoretical description of graphene plasmon scattering at an interface between distinct Fermi levels, which could be relevant for the realization of plasmonic circuitry elements such as plasmonic lenses or reflectors, and for controlling plasmon propagation by modulating the potential landscape of graphene.
\end{abstract}

DOI: 10.1103/PhysRevB.97.035434

\section{INTRODUCTION}

Controlling the propagation of graphene surface plasmon polaritons (SPPs) [1-3] is an important technological problem for applications in SPP circuitry [4,5]. It is well known from elementary wave mechanics that any wave will be both reflected and transmitted at an interface where the properties of the propagating medium change. The situation is no different with graphene SPP in the presence of a spatial change of graphene's conductivity and/or dielectric properties of the surrounding media.

The possibility of generating interfaces for the reflection of graphene SPP by changing graphene's conductivity is particularly attractive for the construction of tunable graphene SPPbased circuitry elements, such as reflectors and beam splitters, due to the possibility of controlling graphene's doping level. In a graphene field effect transistor, the doping of the system is controlled by the gate voltage and by the dielectric between graphene and the gate electrode [6,7]. Therefore, a possible way to create a conductivity interface is to use a graphene field effect transistor with two different dielectric substrates below the graphene layer, as depicted in Fig. 1. Due to the different local capacitances, different electronic densities will be induced in the two graphene regions, which in turn implies a different optical conductivity for the two regions. Another possibility

\footnotetext{
*andrej6@gmail.com

†bruno.a.c.amorim@tecnico.ulisboa.pt; amorim.bac@gmail.com

†bludov@fisica.uminho.pt

Ilpadgo@fotonik.dtu.dk

speres@ fisica.uminho.pt
}

is to consider a single dielectric as the graphene substrate, but using a split gate geometry, such that the applied gate voltage can be independently controlled in two different regions [8]. A spatial modulation of graphene's doping level could also be achieved via nonuniform chemical doping. In general, a graphene SPP incident in a conductivity/dielectric interface will be partially transmitted and partially reflected. Once the problem of plasmon scattering at a single interface is solved, it poses no difficulty to create a SPP filter by combining three different dielectrics in sequence, thereby generalizing the scheme of the device depicted in Fig. 1. It should be noted the scattering of a SPP at an interface involves not only the transmission and reflection of the field as SPP, but also the emission of free radiation $[9,10]$. Ideally, one would want this emission of radiation to be as small as possible in order to keep the energy within the SPP wave. As we shall see ahead, under typical experimental conditions, we predict that the losses in the scattering event via emission of free propagating radiation are minute.

In this paper we study the scattering of a graphene SPP at normal incidence by a conductivity and/or dielectric interface. The scattering problem is treated by expanding the electromagnetic field in terms of a set of local eigenmodes and then using wave matching at the conductivity/dielectric interface. This method takes into account both retardation effects and emission of free radiation. Analytic, approximate expressions are obtained for the graphene SPP reflection and transmission coefficients. The approximate solution is compared to a numerical solution of the wave matching problem. It is worthwhile pointing out that the problem of reflection of graphene SPPs at a conductivity step was previously studied in Ref. [11] employing a fully numerical method, but in the electrostatic 
limit, which does not take into account radiation losses. The problem of reflection at a conductivity interface for non-normal incidence was studied in Ref. [12], also in the electrostatic limit. The scattering of graphene SPPs by a conductivity barrier/well has been considered in Ref. [13], taking into account retardation effects in a fully numerical approach. In addition, the reflection of SPP at a graphene edge was studied in Ref. [14]. Research on graphene plasmonics is a relatively recent topic [1] and research on graphene plasmonic circuitry is still in its infancy. We note, however, that imaging of graphene plasmon scattering on lattice defects $[15,16]$ and corrugations [17] has already been reported. It is also worthwhile noticing that the experimental study of scattering of SPP in metals has also been reported in Refs. [9,18-20] and the generation of unidirection SPP beams was reported in Ref. [21]. On the theoretical side, the problem of scattering of SPP in metals by one-dimensional defects, such as wires or grooves, has been studied in Refs. [4,10,22-25]. Finally, the scattering of phonon polaritons at dielectric interfaces has been studied in Ref. [26].

This paper is organized as follows: In Sec. II we define the problem and lay down the general approach to tackle it based on a local eigenmode expansion of the electromagnetic field and wave matching. We describe the electromagnetic mode structure and dispersion relations, considering graphene SPP, waveguide, and free radiation modes. Section III is devoted to the problem of graphene SPP scattering. In Sec. III A we solve the scattering problem analytically in the approximation of weak coupling of SPPs to radiation modes; in Sec. III B we show that the scattering problem can be recast as a Fredholm equation of the second kind. We show that the approximate results can be recovered from the zeroth order solution of the Fredholm equation in Sec. III B 1. We compare the analytical results with a numeric solution of the Fredholm equation and discuss the obtained results in Sec. IV. Conclusions are drawn in Sec. V.

\section{GEOMETRY AND ELECTROMAGNETIC MODES}

The scattering problem and the geometry we discuss in this paper is represented in Fig. 1. An identical geometry has been considered in the case of scattering of surface phonon polaritons [26]. We assume a plasmon propagating from the left at normal incidence, that is, along the $z$ axis. When impinging at the interface between the dielectrics $\epsilon_{1}$ and $\epsilon_{2}$, part of the plasmon will be reflected, part will be transmitted, and some of the energy will be radiated to the far field. We assume a time dependence of the electromagnetic fields of the form $e^{i \omega t}$.

We obtain the electromagnetic modes of the fields in the geometry depicted in Fig. 1 by solving Maxwell's equations (see Appendix A). The resulting modes are labeled by an index $n$. The properties of these modes are analyzed in detail in this section. We make a piecewise decomposition of the fields in terms of the eigenmodes, using the superscript $<(>)$ for the $z<0(z>0)$ region:

$$
\begin{gathered}
B_{y}^{\lessgtr}(x, z)=\sum_{n, \lambda} \alpha_{n, \lambda}^{\lessgtr} e^{\lambda i q_{n}^{\lessgtr} z} h_{n}^{\lessgtr}(x), \\
E_{x}^{\lessgtr}(x, z)=-\sum_{n, \lambda} \lambda \alpha_{n, \lambda}^{\lessgtr} e^{\lambda i q_{n}^{\lessgtr}} z e_{n}^{\lessgtr}(x),
\end{gathered}
$$

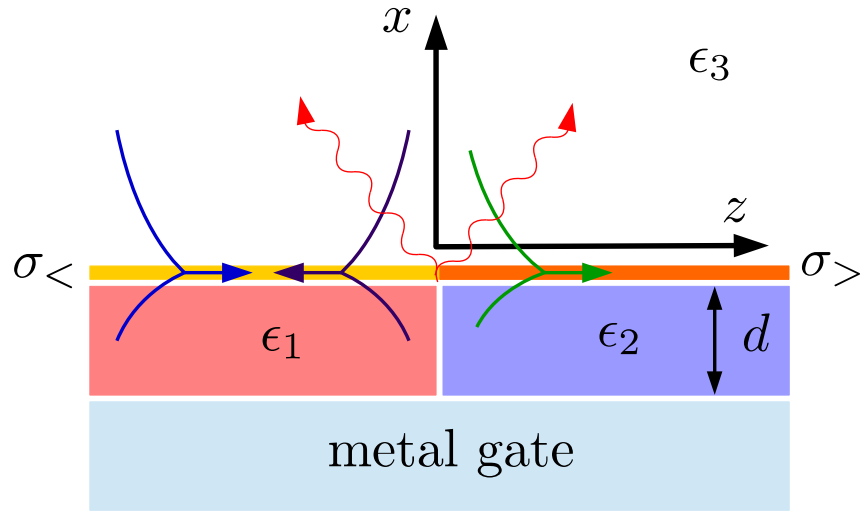

FIG. 1. Illustration of the geometry considered for the SPP scattering problem. The yellow and red lines stand for graphene at two different electronic densities. For simplicity we assume that the electronic density changes abruptly at $z=0$, in a steplike manner. We allow for different dielectric substrates in the regions $z \lessgtr 0$. The presence of a metallic gate allows the tuning of the doping level of the graphene layer. A typical SPP scattering event is represented: A SPP impinging from the left at the interface can both be reflected and transmitted as a SPP or scattered into free radiation.

where $q_{n}^{\lessgtr}$ is the wave number of mode $n$ along the $z$ direction, $\lambda= \pm 1$ indicates a left/right propagating wave, and $\alpha_{n, \lambda}^{\lessgtr}$ are mode amplitudes. We clarify that the sum over $n$ actually denotes a summation over discrete modes and an integration over continuum modes. The eigenmode's $y$ component of the magnetic field reads (the details can be found in Appendix A):

$$
h_{n}^{\lessgtr}(x)=\left\{\begin{array}{ll}
B_{n}^{\lessgtr} e^{p_{3 \mid n}^{\lessgtr} x}+C_{n}^{\lessgtr} e^{-p_{3 \mid n} x}, & x>0 \\
A_{n}^{\lessgtr} \cosh \left[p_{j \mid n}(x+d)\right], & 0>x>-d
\end{array},\right.
$$

where $A_{n}^{\lessgtr}, B_{n}^{\lessgtr}$, and $C_{n}^{\lessgtr}$ are constants to the later defined, the graphene layer is located at $x=0$, and the metallic gate at $x=-d$; we have written $j=1,2$ for the $z<0, z>0$ regions, respectively, and for each region, the wave number along the $x$ direction is given by

$$
\begin{gathered}
\left(p_{1 \mid n}\right)^{2}=\left(q_{n}^{<}\right)^{2}-\epsilon_{1} k_{0}^{2}, \\
\left(p_{2 \mid n}\right)^{2}=\left(q_{n}^{>}\right)^{2}-\epsilon_{2} k_{0}^{2}, \\
\left(p_{3 \mid n}^{\lessgtr}\right)^{2}=\left(q_{n}^{\lessgtr}\right)^{2}-\epsilon_{3} k_{0}^{2},
\end{gathered}
$$

with $k_{0}=\omega / c$ denoting the wave number in vacuum. The relation between the wave number $q_{n}^{\lessgtr}$ and the frequency $\omega$ needs to be calculated for each mode, usually by solving a transcendental equation. In each region $z \lessgtr 0$, the magnetic $h_{n}^{\lessgtr}$ modes can be chosen to satisfy the orthonormality condition (provided there are no losses, i.e., or purely real dielectric functions and a purely imaginary graphene conductivity)

$$
\left\langle h_{n}^{\lessgtr}, e_{m}^{\lessgtr}\right\rangle=\int_{-d}^{\infty} d x h_{n}^{\lessgtr}(x) e_{m}^{\lessgtr}(x)=\delta_{n, m},
$$

where $e_{m}^{\lessgtr}(x)$ gives the $x$ component of the electric field for mode $m$ (see Appendix A). 
From the boundary conditions on the graphene layer, $x=0$, we obtain the following equations for $A_{n}^{\lessgtr}, B_{n}^{\lessgtr}$, and $C_{n}^{\lessgtr}$

$$
\begin{gathered}
B_{n}^{\lessgtr}-C_{n}^{\lessgtr}=\frac{p_{j \mid n} \epsilon_{3}}{p_{3 \mid n}^{\lessgtr} \epsilon_{j}} A_{n}^{\lessgtr} \sinh \left(p_{j \mid n} d\right), \\
B_{n}^{\lessgtr}+C_{n}^{\lessgtr}-A_{n}^{\lessgtr} \cosh \left(p_{j \mid n} d\right)=\frac{\sigma_{\lessgtr}}{i \omega \epsilon_{0}} \frac{p_{j \mid n}}{\epsilon_{j}} A_{n}^{\lessgtr} \sinh \left(p_{j \mid n} d\right) .
\end{gathered}
$$

The solution of the above equations determines the spectrum and the structure of the electromagnetic modes of the system. The wave numbers $p_{j \mid n}$ and $p_{3 \mid n}^{\gtrless}$ can be real or purely imaginary. From these possibilities we can classify the modes as: graphene SPP (both $p_{j \mid n}$ and $p_{3 \mid n}^{\gtrless}$ are real), waveguide modes ( $p_{j \mid n}$ is imaginary and $p_{3 \mid n}^{\gtrless}$ is real), and free radiation modes (both $p_{j \mid n}$ and $p_{3 \mid n}^{\gtrless}$ are imaginary). As we do not want to discuss the decay of the modes as they propagate, but only the scattering event at the abrupt interface, we will neglect dissipative losses. In a physical situation, where the interface is not abrupt, but instead the dielectric constants and the conductivity of the graphene layer change over a finite length $\ell$, neglecting losses in the scattering process should be a good approximation provided the propagation length of the graphene SPP is much greater than $\ell$ [13]. We also point out that even in the presence of losses it is still possible to define orthogonal modes, by suitably defining the inner product [27]. In particular, we neglect the real part of the graphene conductivity, which we model within a Drude model, by approximating $\sigma_{\lessgtr} \simeq i \sigma_{I, \lessgtr}$ where

$$
\sigma_{I, \lessgtr} \simeq-\frac{e^{2}}{\pi \hbar} \frac{E_{F}^{\lessgtr}}{\hbar \omega},
$$

and assume the dielectric constants to be real valued.

\section{A. Graphene SPP}

The graphene SPP is a mode localized in the graphene layer. Further in the paper we will denote the graphene SPP mode by index $n=0$. It is characterized by real $p_{j \mid 0}$ and $p_{3 \mid 0}^{\lessgtr}$. The fact that $p_{3 \mid 0}^{\lessgtr}$ is real forces us to set $B_{0}^{\lessgtr}$ to zero in equations (6) and (7), in order to avoid the unphysical situation of the field growing exponentially when $x \rightarrow+\infty$. This leads to the following implicit condition for the graphene SPP dispersion relation

$$
\frac{\epsilon_{3}}{p_{3 \mid 0}^{\lessgtr}}+\frac{\epsilon_{j}}{p_{j \mid 0}} \operatorname{coth}\left(p_{j \mid 0} d\right)-i \frac{\sigma_{\lessgtr}}{\epsilon_{0} \omega}=0 .
$$

Clearly, when $d \rightarrow \infty$ we recover the dispersion relation of plasmons in a graphene layer clad between two semi-infinite dielectrics [1]. We fix $A_{0}$ by imposing the normalization condition

$$
\left\langle h_{0}^{\lessgtr}, e_{0}^{\lessgtr}\right\rangle=\int_{-d}^{\infty} d x h_{0}^{\lessgtr}(x) e_{0}^{\lessgtr}(x)=1,
$$

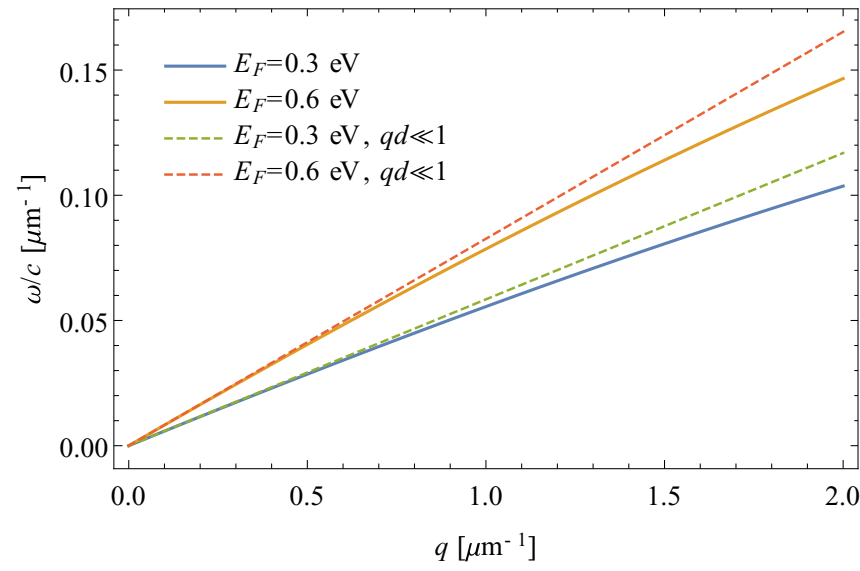

FIG. 2. Dispersion relation of graphene SPP (13) for two different Fermi energies: $E_{F}=0.3 \mathrm{eV}$ (solid blue line) and $E_{F}=0.6 \mathrm{eV}$ (solid yellow line). Also represented are the small wave-number approximations (15) for the plasmon dispersion relation (dashed lines). The values used in the plot are $d=300 \mathrm{~nm}, \epsilon_{j}=3.9$, and $\epsilon_{3}=1$.

leading to

$$
\begin{aligned}
\left(A_{0}^{\lessgtr}\right)^{2}= & \frac{\omega \epsilon_{j}}{4 q_{0}^{\lessgtr} c^{2}}\left(2 d+\frac{\sinh \left(2 p_{j \mid 0} d\right)}{p_{j \mid 0}}\right. \\
& \left.-\frac{\epsilon_{j}}{\epsilon_{3}} \frac{2 \epsilon_{0}^{2} \epsilon_{3}^{2} \omega^{2} \cosh ^{2}\left(p_{j \mid 0} d\right)}{p_{3 \mid 0}^{\lessgtr}\left(p_{3 \mid 0}^{\lessgtr} \sigma_{I, \lessgtr}+\epsilon_{0} \epsilon_{3} \omega\right)^{2}}\right)^{-1} .
\end{aligned}
$$

Approximate solution for graphene SPP dispersion relation. In the electrostatic limit $(c \rightarrow \infty)$, we approximate $p_{j \mid 0} \simeq$ $p_{3 \mid 0}^{\gtrless} \simeq q_{0}^{\gtrless}$. With this approximation equation (9) becomes

$$
\frac{\epsilon_{3}}{q}+\frac{\epsilon_{j}}{q} \operatorname{coth}(q d)-i \frac{\sigma_{\lessgtr}}{\epsilon_{0} \omega}=0 .
$$

Using (8), we can solve the previous equation for $\omega$ obtaining

$$
\hbar \omega=\sqrt{4 \alpha E_{F} \hbar c \frac{q}{\epsilon_{3}+\epsilon_{j} \operatorname{coth}(q d)}},
$$

where we have introduced the fine-structure constant $\alpha=$ $e^{2} /\left(4 \pi \epsilon_{0} c \hbar\right)$. In the limit of a thick substrate $q d \gg 1$, we approximate $\operatorname{coth}(q d) \simeq 1$, recovering the dispersion relation for a surface plasmon polariton in graphene supported by an infinite dielectric

$$
\hbar \omega \simeq \sqrt{4 \alpha E_{F} \hbar c \frac{q}{\epsilon_{3}+\epsilon_{j}}},
$$

with the characteristic $\propto \sqrt{q}$ dependence. In the opposite limit, $q d \ll 1$, we approximate $\operatorname{coth}(q d) \simeq 1 /(q d)$ and obtain

$$
\hbar \omega \simeq \sqrt{4 \alpha E_{F} \hbar c \frac{d}{\epsilon_{j}}} q,
$$

i.e., a linear dispersion relation for small wave numbers.

In Fig. 2 we show the dispersion relation of the SPP for two different Fermi energies. It is clear that for typical substrate thickness and wave numbers, the dispersion relation is closer to linear than to the square root dependence. 


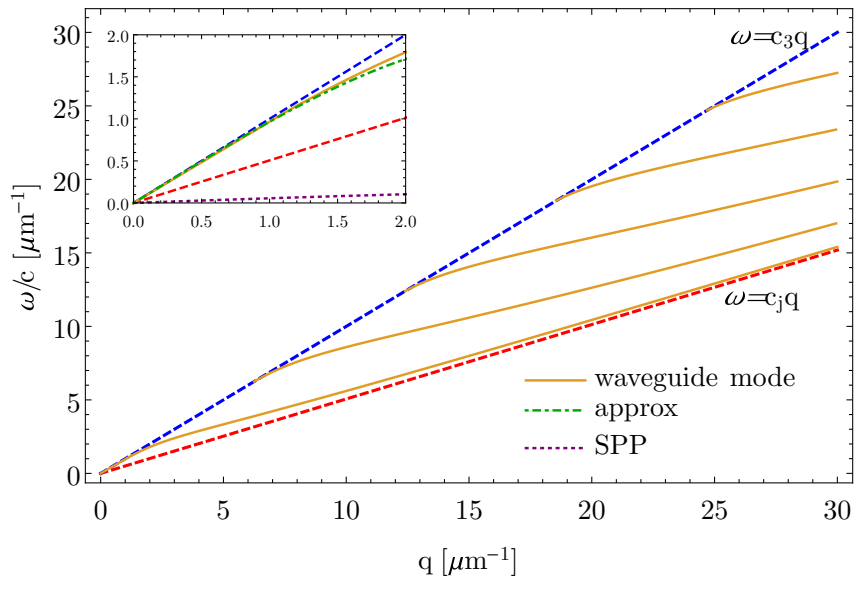

FIG. 3. Dispersion relation, $\omega\left(q_{n}^{<}\right)$, (solid yellow lines) for the first five waveguide modes for a structure with $d=300 \mathrm{~nm}, \epsilon_{j}=3.9$, $\epsilon_{3}=1$, and $E_{F}=0.3 \mathrm{eV}$. The dispersion relation for the waveguide modes in the absence of graphene is indistinguishable from the dispersion shown on the scale used. The light lines $\omega=c_{n} q$ with $c_{n}=c / \sqrt{\epsilon_{n}}$ are shown for $\epsilon_{n}=\epsilon_{3}$ (blue dashed line) and $\epsilon_{n}=\epsilon_{j}$ (red dashed line). Inset: Zoom in the region with $q$ from 0 to $2 \mu \mathrm{m}^{-1}$. The dispersion relation of the graphene surface plasmon polariton is shown by the dotted purple line, and the approximated dispersion relation for the $n=1$ waveguide mode (18) is represented by the dot-dashed green line.

\section{B. Waveguide modes}

In the case where $\epsilon_{j}>\epsilon_{3}$, the structure supports modes which are localized in the region $0>x>-d$, dubbed waveguide modes. Waveguide modes are oscillating in the $0>x>$ $-d$ region, but decay exponentially for $x \rightarrow \infty$. As in the case for graphene SPP, $p_{3 \mid n}^{\gtrless}$ is real and thus we set $B_{n}^{\lessgtr}=0$. However, due to the oscillating nature of the field for $0>x>$ $-d, p_{j \mid n}=i k_{j \mid n}=i \sqrt{\epsilon_{j} k_{0}^{2}-\left(q_{n}^{\lessgtr}\right)^{2}}$ is now purely imaginary. The dispersion relation of the waveguide modes is still given by equation (9), but with imaginary $p_{j \mid n}=i k_{j \mid n}$. Namely, we obtain the condition

$$
\frac{\epsilon_{3}}{p_{3 \mid n}^{\lessgtr}}-\frac{\epsilon_{j}}{k_{j \mid n}} \cot \left(k_{j \mid n} d\right)-i \frac{\sigma_{\lessgtr}}{\epsilon_{0} \omega}=0 .
$$

The solutions for this equation are organized as a series of bands with discrete spectrum, $\omega\left(q_{n}^{\gtrless}\right)$, restricted to the region $c q_{n}^{\gtrless} / \sqrt{\epsilon_{j}}<\omega\left(q_{n}^{\gtrless}\right)<c q_{n}^{\gtrless} / \sqrt{\epsilon_{3}}$, as it is shown in Fig. 3 for a typical setup. As it can be seen from the figure, the lowest, $n=1$, waveguide mode bifurcates from the origin and exists for all positive $\omega$ and $q_{1}^{<}$, while the remaining waveguide modes, $n>1$, bifurcate from the points with frequencies $\omega_{j \mid n}=c(\pi / d)(n-1) / \sqrt{\epsilon_{j} / \epsilon_{3}-1}$, lying on the light line in vacuum $\omega=c q / \sqrt{\epsilon_{3}}$ and existing in the spectral range above those frequencies, $\omega \geqslant \omega_{j \mid n}$. The presence of the graphene has a negligible influence on the spectrum of the waveguide modes for the parameters considered.

Approximate dispersion relation for the lowest waveguide mode. In the limit of small frequency and momentum, and neglecting the effect of the graphene layer, it is possible to obtain an approximate expression for the lowest, $n=1$, waveguide mode dispersion. Neglecting the graphene conductivity term in equation (16) and approximating $\tan \left(k_{j \mid 1} d\right) \simeq k_{j \mid 1} d$, we obtain the following condition

$$
1=\frac{\epsilon_{3} k_{j \mid 1}^{2} d}{\epsilon_{j} p_{3 \mid 1}^{\lessgtr}} .
$$

Recalling the definitions of $k_{j \mid 1}=\sqrt{\epsilon_{j} k_{0}^{2}-q_{1}^{\lessgtr 2}}$ and $p_{3 \mid 1}^{\lessgtr}=$ $\sqrt{q_{1}^{\lessgtr 2}-\epsilon_{3} k_{0}^{2}}$, the previous equation can be solved to lowest order in $q_{1}^{\lessgtr}$, leading to the approximate dispersion relation for the $n=1$ waveguide mode

$$
\omega\left(q_{1}^{\lessgtr}\right) \simeq \frac{c q_{1}^{\lessgtr}}{\sqrt{\epsilon_{3}}} \sqrt{1-\left(q_{1}^{\lessgtr} d\right)^{2}\left(\frac{\epsilon_{j}-\epsilon_{3}}{\epsilon_{j}}\right)^{2}} .
$$

This approximate expression for the dispersion relation of the lowest waveguide mode is shown in Fig. 3. It is clearly seen that for the parameters of Fig. 3 this approximation is valid for $q_{1}^{\lessgtr} \lesssim 1.5 \mu \mathrm{m}^{-1}$ and fails for larger wave numbers.

\section{Radiative modes}

Besides localized modes (SPP and waveguide), there is a continuum of radiative modes. Radiative modes are characterized by $\epsilon_{3} k_{0}^{2}, \epsilon_{j} k_{0}^{2}>\left(q_{n}\right)^{2}$. We chose to label these modes by their frequency $\omega$ and momentum along the $x$ direction in the region $x>0, k$, which we can choose to be positive, such that $p_{3 \mid k}^{\gtrless}=i k$. In this situation, we obtain

$$
q_{k}^{\lessgtr}=\sqrt{\epsilon_{3} k_{0}^{2}-k^{2}},
$$

and substituting this in (4) gives us

$$
p_{j \mid k}=\sqrt{\left(\epsilon_{3}-\epsilon_{j}\right) k_{0}^{2}-k^{2}},
$$

where we have substituted the index $n$ by $k$. Equation (19) corresponds to the dispersion relation of the radiative modes. The aforementioned positiveness of $k$ results in the fact that the dispersion relation of these modes lies above the light line for a dielectric with $\epsilon_{3}$ (see Fig. 3). Notice that for $k^{2}>k_{c}^{2}=\epsilon_{3} k_{0}^{2}$ the radiative modes are actually evanescent waves along the $z$ direction with imaginary $q_{k}^{\lessgtr}$. Therefore, it is with some abuse of language that we refer to them as radiation modes. On the other hand, for $k^{2}<k_{c}^{2}, q_{k}^{\lessgtr}$ is real and we wave a true radiation mode corresponding to a propagating wave in both the $x$ and $z$ directions. Both kinds of modes are necessary when making the mode matching at the interface $z=0$. We also have that $p_{j \mid k}$ is real for $k^{2}<\left(\epsilon_{3}-\epsilon_{j}\right) k_{0}^{2}$ [see equation (20)], thus describing evanescent waves along the $x$ direction, in the substrate with dielectric constant $\epsilon_{j}$, and is imaginary in the opposite situation $k^{2}>\left(\epsilon_{3}-\epsilon_{j}\right) k_{0}^{2}$, which corresponds to the propagating wave along the $x$ direction in the substrate (when $\epsilon_{j}>\epsilon_{3}$ waves for any $k$ are of that type). For radiation modes all the coefficients $A_{k}^{\lessgtr}, B_{k}^{\lessgtr}$, and $C_{k}^{\lessgtr}$ in equation (3) are nonzero. Imposing the boundary conditions at the $x=0$ interface (see Appendix A) we can write $B_{k}^{\lessgtr}$ and $C_{k}^{\lessgtr}$ as

$$
\begin{aligned}
& B_{k}^{\lessgtr}=\frac{A_{k}^{\lessgtr}}{2}\left(\mathcal{F}_{k}^{\lessgtr}-i \mathcal{G}_{k}^{\lessgtr}\right), \\
& C_{k}^{\lessgtr}=\frac{A_{k}^{\lessgtr}}{2}\left(\mathcal{F}_{k}^{\lessgtr}+i \mathcal{G}_{k}^{\lessgtr}\right),
\end{aligned}
$$


where we have defined

$$
\begin{gathered}
\mathcal{F}_{k}^{\lessgtr}=\cosh \left(p_{j \mid k} d\right)+\frac{\sigma_{I, \lessgtr}}{\omega \epsilon_{0}} \frac{p_{j \mid k}}{\epsilon_{j}} \sinh \left(p_{j \mid k} d\right), \\
\mathcal{G}_{k}^{\lessgtr}=\frac{p_{j \mid k} \epsilon_{3}}{k \epsilon_{j}} \sinh \left(p_{j \mid k} d\right) .
\end{gathered}
$$

The electric and magnetic field modes, can thus be written as

$$
\begin{aligned}
& h_{k}^{\lessgtr}(x) \\
& \quad=A_{k}^{\lessgtr} \frac{q_{k}^{\lessgtr} c^{2}}{\omega \epsilon_{3}} \begin{cases}\mathcal{F}_{k}^{\lessgtr} \cos (k x)+\mathcal{G}_{k}^{\lessgtr} \sin (k x), & x>0 \\
\cosh \left[p_{j \mid k}(x+d)\right], & 0>x>-d\end{cases}
\end{aligned}
$$

and the corresponding $x$ component of the electric field reads

$$
\begin{aligned}
& e_{k}^{\lessgtr}(x) \\
& \quad=A_{k}^{\lessgtr} \frac{q_{k}^{\lessgtr} c^{2}}{\omega \epsilon_{3}}\left\{\begin{array}{ll}
\mathcal{F}_{k}^{\lessgtr} \cos (k x)+\mathcal{G}_{k}^{\lessgtr} \sin (k x), & x>0 \\
\frac{\epsilon_{3}}{\epsilon_{j}} \cosh \left[p_{j \mid k}(x+d)\right], & 0>x>-d
\end{array} .\right.
\end{aligned}
$$

The modes can be normalized through the condition:

$$
\int_{-d}^{\infty} d x h_{k}^{\lessgtr}(x) e_{k^{\prime}}^{\lessgtr}(x)=\delta\left(k-k^{\prime}\right),
$$

which fixes $A_{k}^{\lessgtr}$ to have the value

$$
\left(A_{k}^{\lessgtr}\right)^{2}=\frac{\omega \epsilon_{3}}{q_{k}^{\lessgtr} c^{2}} \frac{2}{\pi} \frac{1}{\left|\mathcal{F}_{k}^{\lessgtr}\right|^{2}+\left|\mathcal{G}_{k}^{\lessgtr}\right|^{2}} .
$$

Notice that $A_{k}^{\lessgtr}$ will be imaginary when $q_{k}^{\lessgtr}$ is imaginary.

\section{SPP SCATTERING}

We now consider the problem of scattering of a graphene SPP which is illustrated in Fig. 1. A plasmon coming from the left and impinging at the dielectric/conductivity interface at $z=0$ is scattered into both a back-scattered (reflected) and forward-scattered (transmitted) plasmon, and also into free propagating radiation. For simplicity, we will consider a situation where no waveguide modes are supported $\left(\epsilon_{j}<\epsilon_{3}\right)$. In order to determine the total field in the regions $z \lessgtr 0$, we must consider both the discrete plasmon mode and the radiative modes. Therefore the expansion of the electric and magnetic fields in terms of local eigenmodes, equations (1) and (2), reads for $z<0$ (note the phase of $\pi$ introduced in the reflection coefficients of the electric field)

$$
\begin{aligned}
E_{x}^{<}(x, z)= & e_{0}^{<}(x) e^{-i q_{0}^{<} z}-e_{0}^{<}(x) r_{0} e^{i q_{0}^{<} z} \\
& -\int_{0}^{\infty} d k r_{k} e_{k}^{<}(x) e^{i q_{k}^{<} z}, \\
B_{y}^{<}(x, z)= & h_{0}^{<}(x) e^{-i q_{0}^{<} z}+h_{0}^{<}(x) r_{0} e^{i q_{0}^{<} z} \\
& +\int_{0}^{\infty} d k r_{k} h_{k}^{<}(x) e^{i q_{k}^{<} z},
\end{aligned}
$$

while for $z>0$ we write

$$
\begin{aligned}
& E_{x}^{>}(x, z)=e_{0}^{>}(x) \tau_{0} e^{-i q_{0}^{>} z}+\int_{0}^{\infty} d k \tau_{k} e_{k}^{>}(x) e^{-i q_{k}^{>} z}, \\
& B_{y}^{>}(x, z)=h_{0}^{>}(x) \tau_{0} e^{-i q_{0}^{>} z}+\int_{0}^{\infty} d k \tau_{k} h_{k}^{>}(x) e^{-i q_{k}^{>} z} .
\end{aligned}
$$

In these expressions, $r_{0} / \tau_{0}$ and $r_{k} / \tau_{k}$ are, respectively, the reflection/transmission amplitudes for the SPP and radiative modes with wave number $k$ along the $x$ direction, for $x>0$. The relation between the frequency $\omega$ and the in-plane graphene SPP momentum, $q_{0}^{\lessgtr}$, is determined by equation (13).

Performing mode matching by enforcing the continuity of $E_{x}(x, z)$ and $B_{y}(x, z)$ at $z=0$, we obtain the set of equations

$$
\begin{array}{r}
e_{0}^{<}(x)\left(1-r_{0}\right)-\int_{0}^{\infty} d k r_{k} e_{k}^{<}(x) \\
=\tau_{0} e_{0}^{>}(x)+\int_{0}^{\infty} d k \tau_{k} e_{k}^{>}(x), \\
h_{0}^{<}(x)\left(1+r_{0}\right)+\int_{0}^{\infty} d k r_{k} h_{k}^{<}(x) \\
=\tau_{0} h_{0}^{>}(x)+\int_{0}^{\infty} d k \tau_{k} h_{k}^{<}(x) .
\end{array}
$$

Note that in order to satisfy the matching conditions at $z=0$, we need both propagating and evanescent radiative modes along the $z$ direction. To determine the reflection and transmission amplitudes, we take the inner product [as defined in (5)] of (33) with $h_{0}^{>}(x)$ and $h_{k}^{>}(x)$, and the inner product of (34) with $e_{0}^{>}(x)$ and $e_{k}^{>}(x)$. Using the orthonormality of the modes, we obtain the following system of equations

$$
\begin{aligned}
& \tau_{0}=\left(1-r_{0}\right)\left\langle h_{0}^{>}, e_{0}^{<}\right\rangle-\int_{0}^{\infty} d k r_{k}\left\langle h_{0}^{>}, e_{k}^{<}\right\rangle, \\
& \tau_{0}=\left(1+r_{0}\right)\left\langle e_{0}^{>}, h_{0}^{<}\right\rangle+\int_{0}^{\infty} d k r_{k}\left\langle e_{0}^{>}, h_{k}^{<}\right\rangle,
\end{aligned}
$$

and

$$
\begin{aligned}
& \tau_{k}=\left(1-r_{0}\right)\left\langle h_{k}^{>}, e_{0}^{<}\right\rangle-\int_{0}^{\infty} d k^{\prime} r_{k^{\prime}}\left\langle h_{k}^{>}, e_{k^{\prime}}^{<}\right\rangle, \\
& \tau_{k}=\left(1+r_{0}\right)\left\langle e_{k}^{>}, h_{0}^{<}\right\rangle+\int_{0}^{\infty} d k^{\prime} r_{k^{\prime}}\left\langle e_{k}^{>}, h_{k^{\prime}}^{<}\right\rangle .
\end{aligned}
$$

The solution of this system of coupled integral equations yields the reflection and transmission amplitudes. In the following, we will provide both an approximate analytic solution and a full numerical solution for this system of equations.

\section{A. Approximate analytical solution}

In order to proceed analytically, we will introduce some approximations. We assume that the following relations hold [26]

$$
\begin{gathered}
\left\langle h_{0}^{>}, e_{k}^{<}\right\rangle \simeq\left\langle e_{0}^{>}, h_{k}^{<}\right\rangle \simeq\left\langle h_{k}^{>}, e_{0}^{<}\right\rangle \simeq\left\langle e_{k}^{>}, h_{0}^{<}\right\rangle \simeq 0, \\
\left\langle h_{k}^{>}, e_{k^{\prime}}^{<}\right\rangle \simeq \delta\left(k-k^{\prime}\right) .
\end{gathered}
$$

Mathematically these relations mean that the modes of the different regions are almost orthogonal. Physically, we can understand this as a statement that the SPP modes are weakly 


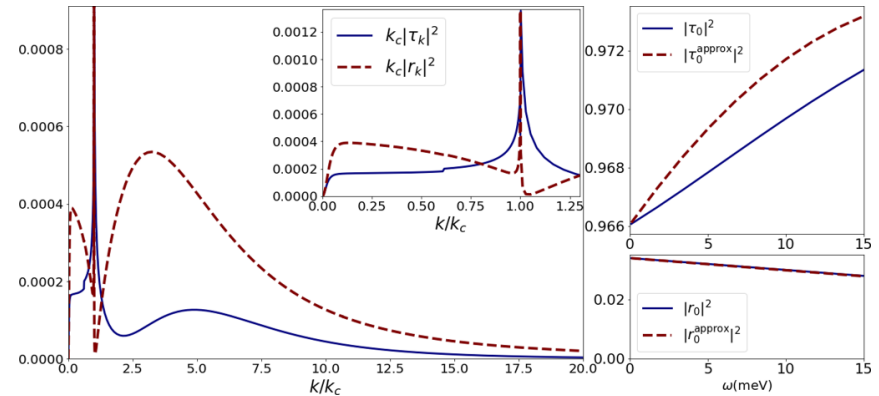

FIG. 4. Left panel: Transmittance and reflectance of the radiative modes as a function of $k / k_{c}$, in the interval $k \in\left[0,20 k_{c}\right]$, for $\omega=15$ $\mathrm{meV}$. The inset zooms in the interval $k \in\left[0,1.25 k_{c}\right]$. Right panel: Transmittance (top right) and reflectance (bottom left) of graphene SPP as a function of the plasmon frequency. Both the results obtained with the analytic approximation (dashed purple) and the full numerical solution (solid blue) are represented. The difference between the numeric solution of Fredholm equation and the approximated solution for the reflection and transmission coefficients is smaller than $1 \%$. In both panels the used parameters are: $\mathrm{meV}, d=300 \mathrm{~nm}, \epsilon_{1}=1.5$, $\epsilon_{2}=2.5, \epsilon_{3}=4, E_{F}^{<}=0.37 \mathrm{eV}, E_{F}^{>}=0.47$.

coupled to the radiation modes. The previous relations are approximately true as long as $\epsilon_{1} \simeq \epsilon_{2}$ and $E_{F}^{<} \simeq E_{F}^{>}$. This regime implies small reflection amplitudes, as can be seen in Fig. 4. However, as we will see below, the approximation performs well even beyond this regime. With the aforementioned approximations, equations (35) and (36) become

$$
\begin{aligned}
& \tau_{0}^{\text {approx }}=\left(1-r_{0}^{\text {approx }}\right)\left\langle h_{0}^{>}, e_{0}^{<}\right\rangle, \\
& \tau_{0}^{\text {approx }}=\left(1+r_{0}^{\text {approx }}\right)\left\langle e_{0}^{>}, h_{0}^{<}\right\rangle .
\end{aligned}
$$

We have thus obtained a closed set of two equations for the SPP reflection and transmission coefficients. Solving these, we obtain

$$
\begin{aligned}
r_{0}^{\text {approx }} & =\frac{\left\langle h_{0}^{>}, e_{0}^{<}\right\rangle-\left\langle e_{0}^{>}, h_{0}^{<}\right\rangle}{\left\langle h_{0}^{>}, e_{0}^{<}\right\rangle+\left\langle e_{0}^{>}, h_{0}^{<}\right\rangle}, \\
\tau_{0}^{\text {approx }} & =2 \frac{\left\langle h_{0}^{>}, e_{0}^{<}\right\rangle\left\langle e_{0}^{>}, h_{0}^{<}\right\rangle}{\left\langle h_{0}^{>}, e_{0}^{<}\right\rangle+\left\langle e_{0}^{>}, h_{0}^{<}\right\rangle} .
\end{aligned}
$$

The transmission and reflection coefficients for the radiative modes can be obtained from equations (37) and (38) if we use the approximation (40), while keeping $\left\langle h_{k}^{>}, e_{0}^{<}\right\rangle$and $\left\langle e_{k}^{>}, h_{0}^{<}\right\rangle$ (in order to obtain a nonzero result). We obtain the following equations

$$
\begin{aligned}
\tau_{k}^{\text {approx }} & =\left(1-r_{0}^{\text {approx }}\right)\left\langle h_{k}^{>}, e_{0}^{<}\right\rangle-r_{k}^{\text {approx }}, \\
\tau_{k}^{\text {approx }} & =\left(1+r_{0}^{\text {approx }}\right)\left\langle e_{k}^{>}, h_{0}^{<}\right\rangle+r_{k}^{\text {approx }} .
\end{aligned}
$$

Using the previously obtained value for $r_{0}$, we can solve for $r_{k}$ and $\tau_{k}$, yielding

$$
\begin{aligned}
r_{k}^{\text {approx }} & =\frac{\left\langle e_{0}^{>}, h_{0}^{<}\right\rangle\left\langle h_{k}^{>}, e_{0}^{<}\right\rangle-\left\langle h_{0}^{>}, e_{0}^{<}\right\rangle\left\langle e_{k}^{>}, h_{0}^{<}\right\rangle}{\left\langle h_{0}^{>}, e_{0}^{<}\right\rangle+\left\langle e_{0}^{>}, h_{0}^{<}\right\rangle}, \\
\tau_{k}^{\text {approx }} & =\frac{\left\langle e_{0}^{>}, h_{0}^{<}\right\rangle\left\langle h_{k}^{>}, e_{0}^{<}\right\rangle+\left\langle h_{0}^{>}, e_{0}^{<}\right\rangle\left\langle e_{k}^{>}, h_{0}^{<}\right\rangle}{\left\langle h_{0}^{>}, e_{0}^{<}\right\rangle+\left\langle e_{0}^{>}, h_{0}^{<}\right\rangle} .
\end{aligned}
$$

The inner products in the above equations can be computed analytically and explicit expressions are given in Appendix C.
One comment regarding the validity of the employed approximations is in order. Notice that instead of contracting equations (33) and (34) with $h_{0}^{>}(x)$ and $e_{0}^{>}(x)$, as done to obtain equations (35) and (36), we could have contracted them with $h_{0}^{<}(x)$ and $e_{0}^{<}(x)$. Such a procedure would lead to the following equations

$$
\begin{aligned}
& 1-r_{0}=\tau_{0}\left\langle h_{0}^{<}, e_{0}^{>}\right\rangle+\int_{0}^{\infty} d k \tau_{k}\left\langle h_{0}^{<}, e_{k}^{>}\right\rangle, \\
& 1+r_{0}=\tau_{0}\left\langle e_{0}^{<}, h_{0}^{>}\right\rangle+\int_{0}^{\infty} d k \tau_{k}\left\langle e_{0}^{<}, h_{k}^{<}\right\rangle .
\end{aligned}
$$

Using the approximations (39) and (40), we obtain

$$
\begin{aligned}
& 1-r_{0}^{\text {approx }^{\prime}}=\tau_{0}^{\text {approx }^{\prime}}\left\langle h_{0}^{<}, e_{0}^{>}\right\rangle, \\
& 1+r_{0}^{\text {approx }^{\prime}}=\tau_{0}^{\text {approx }}\left\langle e_{0}^{<}, h_{0}^{>}\right\rangle .
\end{aligned}
$$

Solving these equations gives us the alternative expressions for the reflection and transmission coefficients

$$
\begin{aligned}
r_{0}^{\text {approx }^{\prime}} & =\frac{\left\langle e_{0}^{<}, h_{0}^{>}\right\rangle-\left\langle h_{0}^{<}, e_{0}^{>}\right\rangle}{\left\langle e_{0}^{<}, h_{0}^{>}\right\rangle+\left\langle h_{0}^{<}, e_{0}^{>}\right\rangle}, \\
\tau_{0}^{\text {approx }} & =\frac{2}{\left\langle e_{0}^{<}, h_{0}^{>}\right\rangle+\left\langle h_{0}^{<}, e_{0}^{>}\right\rangle} .
\end{aligned}
$$

Since $e_{0}^{\lessgtr}$ and $h_{0}^{\lessgtr}$ can be chosen as real, we conclude that equations (43) and (53) for $r_{0}$ coincide. However, we see that equations (44) and (54) differ by a factor of $\left\langle h_{0}^{>}, e_{0}^{<}\right\rangle\left\langle e_{0}^{>}, h_{0}^{<}\right\rangle$. This gives us an internal consistency check for the employed approximations: They remain valid as long as

$$
\left\langle h_{0}^{>}, e_{0}^{<}\right\rangle\left\langle e_{0}^{>}, h_{0}^{<}\right\rangle \simeq 1,
$$

which implies a strong coupling between the SPP modes from $z<0$ and for $z>0$.

Note that the value for $r_{0}$ obtained with these approximations is purely real. Therefore there is no phase shift in the back-scattering amplitude of the plasmon, except for the already included phase-shift of $\pi$. This is a consequence of the approximation introduced above and contrasts with the results of Refs. [11,14], obtained within the electrostatic limit, thus ignoring retardation effects.

It should also be noted that the formalism is capable of describing the reflection of a graphene plasmon at the edge of a semi-infinite graphene sheet. We have verified numerically that in this case the transmittance is numerically very small (due to the approximation not being exactly zero) and the reflectance is essentially equal to unity (results not shown; numerically we take the Fermi energy at the right of $z=0$ a very small number, typically $E_{F}^{>} \sim 10^{-3} E_{F}^{<}$, as the numerical procedure does not allow a zero Fermi energy).

In Ref. [11], an electrostatic calculation predicts that the reflection coefficient for graphene in vacuum and subject to a conductivity step at $z=0$ is given by

$$
\left|r_{0}\right|^{2}=\left(\frac{q_{0}^{<}-q_{0}^{>}}{q_{0}^{<}+q_{0}^{>}}\right)^{2} .
$$

If we use the numbers of Fig. 4 for the Fermi energies and plug in the corresponding wave vectors in equation (56) we obtain the value $\left|r_{0}\right|^{2} \approx 0.049$, whereas our calculation in the same 
conditions predicts a value in the range $\left|r_{0}\right|^{2} \approx 0.049-0.016$, as the frequency of the incoming SPP ranges from zero to $\sim 16 \mathrm{meV}$. Note that a consequence of the electrostatic approximation is that the reflection coefficient becomes frequency independent. When taking the electrostatic limit, we can study two possible cases: (i) thin substrate limit, $d \rightarrow 0$, and (ii) thick substrate limit, $d \rightarrow \infty$.

In the electrostatic and thin substrate limits $(\omega / c, d \rightarrow 0)$ the reflectance amplitude (43) reads

$$
r_{0}=\frac{\epsilon_{2} q_{0}^{<}-\epsilon_{1} q_{0}^{>}}{\epsilon_{2} q_{0}^{<}+\epsilon_{1} q_{0}^{>}}
$$

in agreement with the result of Ref. [11] for $\epsilon_{1}=\epsilon_{2}$. For the transmittance amplitude (44), and in the same limit as before, we obtain

$$
\tau_{0}=\frac{2 \sqrt{q_{0}^{>} q_{0}^{<} \epsilon_{1} \epsilon_{2}}}{\epsilon_{2} q_{0}^{<}+\epsilon_{1} q_{0}^{>}} .
$$

Physically, the limit $d \rightarrow 0$ means that the plasmon fields are finite only in the dielectric $\epsilon_{3}$, as the field is screened by the metallic gate. We also note that equations (57) and (58) contain the limit of total reflection when $q_{0}^{<} \rightarrow 0$. As anticipated, it is possible to have SPP reflection even if $E_{F}^{<}=E_{F}^{>}$, provided that $\epsilon_{1}$ and $\epsilon_{2}$ differ.

Conversely, in the electrostatic and thick substrate limits $(\omega / c \rightarrow 0, d \rightarrow \infty)$, we obtain for $r_{0}(43)$ and $\tau_{0}(44)$

$$
\begin{gathered}
r_{0}=\frac{\left(\epsilon_{2}+\epsilon_{3}\right) q_{0}^{<}-\left(\epsilon_{1}+\epsilon_{3}\right) q_{0}^{>}}{\left(\epsilon_{2}+\epsilon_{3}\right) q_{0}^{<}+\left(\epsilon_{1}+\epsilon_{3}\right) q_{0}^{>}}, \\
\tau_{0}=\frac{4 q_{0}^{<} q_{0}^{>} \sqrt{\left(\epsilon_{1}+\epsilon_{3}\right)\left(\epsilon_{2}+\epsilon_{3}\right)}}{\left(q_{0}^{<}+q_{0}^{>}\right)\left[\left(\epsilon_{2}+\epsilon_{3}\right) q_{0}^{<}+\left(\epsilon_{1}+\epsilon_{3}\right) q_{0}^{>}\right]} .
\end{gathered}
$$

\section{B. Formulation as a Fredholm equation}

We will now recast the scattering problem in a form amiable to a numerical solution. While doing that, we will see how the approximate analytic result corresponds to a lowest order approximation to the solution of the complete problem.

Recalling equations (35)-(38) and subtracting equation (36) from equation (35), we obtain

$$
r_{0}=\frac{\left\langle h_{0}^{>}, e_{0}^{<}\right\rangle-\left\langle e_{0}^{>}, h_{0}^{<}\right\rangle}{\left\langle h_{0}^{>}, e_{0}^{<}\right\rangle+\left\langle e_{0}^{>}, h_{0}^{<}\right\rangle}-\int_{0}^{\infty} d k \frac{\left\langle h_{0}^{>}, e_{k}^{<}\right\rangle+\left\langle e_{0}^{>}, h_{k}^{<}\right\rangle}{\left\langle h_{0}^{>}, e_{0}^{<}\right\rangle+\left\langle e_{0}^{>}, h_{0}^{<}\right\rangle} r_{k} .
$$

Furthermore, subtracting equation (38) from equation (37), we obtain

$r_{0}=\frac{\left\langle h_{k}^{>}, e_{0}^{<}\right\rangle-\left\langle e_{k}^{>}, h_{0}^{<}\right\rangle}{\left\langle h_{k}^{>}, e_{0}^{<}\right\rangle+\left\langle e_{k}^{>}, h_{0}^{<}\right\rangle}-\int_{0}^{\infty} d k^{\prime} \frac{\left\langle h_{k}^{>}, e_{k^{\prime}}^{<}\right\rangle+\left\langle e_{k}^{>}, h_{k^{\prime}}^{<}\right\rangle}{\left\langle h_{k}^{>}, e_{0}^{<}\right\rangle+\left\langle e_{k}^{>}, h_{0}^{<}\right\rangle} r_{k^{\prime}}$.

Combining equations (61) and (62) we eliminate $r_{0}$ and obtain a closed equation for the reflection coefficients $r_{k}$

$$
z_{1}(k)+\int_{0}^{\infty} d k^{\prime} z_{2}\left(k, k^{\prime}\right) r_{k^{\prime}}=0
$$

where we have introduced the quantities

$$
\begin{aligned}
& z_{1}(k)=\frac{\left\langle h_{0}^{>}, e_{0}^{<}\right\rangle-\left\langle e_{0}^{>}, h_{0}^{<}\right\rangle}{\left\langle h_{0}^{>}, e_{0}^{<}\right\rangle+\left\langle e_{0}^{>}, h_{0}^{<}\right\rangle}-\frac{\left\langle h_{k}^{>}, e_{0}^{<}\right\rangle-\left\langle e_{k}^{>}, h_{0}^{<}\right\rangle}{\left\langle h_{k}^{>}, e_{0}^{<}\right\rangle+\left\langle e_{k}^{>}, h_{0}^{<}\right\rangle}, \\
& z_{2}\left(k, k^{\prime}\right)=\frac{\left\langle h_{k}^{>}, e_{k^{\prime}}^{<}\right\rangle+\left\langle e_{k}^{>}, h_{k^{\prime}}^{<}\right\rangle}{\left\langle h_{k}^{>}, e_{0}^{<}\right\rangle+\left\langle e_{k}^{>}, h_{0}^{<}\right\rangle}-\frac{\left\langle h_{0}^{>}, e_{k^{\prime}}^{<}\right\rangle+\left\langle e_{0}^{>}, h_{k^{\prime}}^{<}\right\rangle}{\left\langle h_{0}^{>}, e_{0}^{<}\right\rangle+\left\langle e_{0}^{>}, h_{0}^{<}\right\rangle} .
\end{aligned}
$$

Equation (63) is in the form of a Fredholm integral equation of the first kind. However, as shown in Appendix C, the integration kernel $z_{2}\left(k, k^{\prime}\right)$ contains a term that is proportional to a $\delta$ function [see equations (C12) and (C13)]. Therefore, we can split $z_{2}\left(k, k^{\prime}\right)$ as

$$
z_{2}\left(k, k^{\prime}\right)=v(k) \delta\left(k-k^{\prime}\right)+v(k) z_{3}\left(k, k^{\prime}\right),
$$

where $v(k)$ is the diagonal part of $z_{2}\left(k, k^{\prime}\right)$, with its explicit form given in equation (C25), and we have written the remaining part as $v(k) z_{3}\left(k, k^{\prime}\right)$. Inserting this equation into equation (63) and using the $\delta$ function to perform the integration over $k^{\prime}$, we can transform the problem into a Fredholm integral equation of the second kind, as

$$
r_{k}=-\frac{z_{1}(k)}{v(k)}-\int_{0}^{\infty} d k^{\prime} z_{3}\left(k, k^{\prime}\right) r_{k^{\prime}}
$$

This equation can be solved numerically, by discretizing the integral over $k^{\prime}$ using a Gaussian quadrature method, and evaluating the equation for values of $k$ on that same discretized grid, reducing the integral equation to a problem of linear algebra as described in greater detail in Appendix D.

Having obtained the reflection coefficient $r_{k}$, the reflection coefficient for the SPP mode $r_{0}$ can be computed from equation (61). With the knowledge of all the reflection coefficients, the transmission coefficient $\tau_{0}$ can be calculated from equation (35) as

$$
\begin{aligned}
\tau_{0}= & 2 \frac{\left\langle h_{0}^{>}, e_{0}^{<}\right\rangle\left\langle e_{0}^{>}, h_{0}^{<}\right\rangle}{\left\langle h_{0}^{>}, e_{0}^{<}\right\rangle+\left\langle e_{0}^{>}, h_{0}^{<}\right\rangle} \\
& +\int_{0}^{\infty} d k \frac{\left\langle h_{0}^{>}, e_{0}^{<}\right\rangle\left\langle e_{0}^{>}, h_{k}^{<}\right\rangle-\left\langle h_{0}^{>}, e_{k}^{<}\right\rangle\left\langle e_{0}^{>}, h_{0}^{<}\right\rangle}{\left\langle h_{0}^{>}, e_{0}^{<}\right\rangle+\left\langle e_{0}^{>}, h_{0}^{<}\right\rangle} r_{k},
\end{aligned}
$$

and the transmission coefficients $\tau_{k}$ can be determined from equations (61) and (38) as

$$
\begin{aligned}
\tau_{k}= & \frac{\left\langle h_{0}^{>}, e_{0}^{<}\right\rangle\left\langle e_{k}^{>}, h_{0}^{<}\right\rangle+\left\langle e_{0}^{>}, h_{0}^{<}\right\rangle\left\langle h_{k}^{>}, e_{0}^{<}\right\rangle}{\left\langle h_{0}^{>}, e_{0}^{<}\right\rangle+\left\langle e_{0}^{>}, h_{0}^{<}\right\rangle} \\
& +\int_{0}^{\infty} d k^{\prime}\left[\left\langle e_{k}^{>}, h_{k^{\prime}}^{<}\right\rangle-\frac{\left\langle h_{0}^{>}, e_{k^{\prime}}^{<}\right\rangle+\left\langle e_{0}^{>}, h_{k^{\prime}}^{<}\right\rangle}{\left\langle h_{0}^{>}, e_{0}^{<}\right\rangle+\left\langle e_{0}^{>}, h_{0}^{<}\right\rangle}\left\langle e_{k}^{>}, h_{0}^{<}\right\rangle\right] r_{k^{\prime}} \\
& -\frac{\left\langle e_{0}^{>}, h_{0}^{<}\right\rangle\left\langle h_{k}^{>}, e_{0}^{<}\right\rangle-\left\langle h_{0}^{>}, e_{0}^{<}\right\rangle\left\langle e_{k}^{>}, h_{0}^{<}\right\rangle}{\left\langle h_{0}^{>}, e_{0}^{<}\right\rangle+\left\langle e_{0}^{>}, h_{0}^{<}\right\rangle} .
\end{aligned}
$$

This provides a general scheme to fully solve the scattering problem. 
Notice that equations (61), (68), and (69) can be rewritten as

$$
\begin{gathered}
r_{0}=r_{0}^{\text {approx }}-\int_{0}^{\infty} d k \frac{\left\langle h_{0}^{>}, e_{k}^{<}\right\rangle+\left\langle e_{0}^{>}, h_{k}^{<}\right\rangle}{\left\langle h_{0}^{>}, e_{0}^{<}\right\rangle+\left\langle e_{0}^{>}, h_{0}^{<}\right\rangle} r_{k}, \\
\tau_{0}=\tau_{0}^{\text {approx }} \\
+\int_{0}^{\infty} d k \frac{\left\langle h_{0}^{>}, e_{0}^{<}\right\rangle\left\langle e_{0}^{>}, h_{k}^{<}\right\rangle-\left\langle h_{0}^{>}, e_{k}^{<}\right\rangle\left\langle e_{0}^{>}, h_{0}^{<}\right\rangle}{\left\langle h_{0}^{>}, e_{0}^{<}\right\rangle+\left\langle e_{0}^{>}, h_{0}^{<}\right\rangle} r_{k}, \\
\tau_{k}=\tau_{k}^{\text {approx }}-r_{k}^{\text {approx }} \\
+\int_{0}^{\infty} d k^{\prime}\left[\left\langle e_{k}^{>}, h_{k^{\prime}}^{<}\right\rangle-\frac{\left\langle h_{0}^{>}, e_{k^{\prime}}^{<}\right\rangle+\left\langle e_{0}^{>}, h_{k^{\prime}}^{<}\right\rangle}{\left\langle h_{0}^{>}, e_{0}^{<}\right\rangle+\left\langle e_{0}^{>}, h_{0}^{<}\right\rangle}\left\langle e_{k}^{>}, h_{0}^{<}\right\rangle\right] r_{k^{\prime}},
\end{gathered}
$$

with $r_{0}^{\text {approx }}, \tau_{0}^{\text {approx }}, r_{k}^{\text {approx }}$, and $\tau_{k}^{\text {approx }}$ the analytical approximate results given, respectively, by equations (43), (44), (47), and (48). In the following, we will see how the approximate analytic result from Sec. III A can be recovered from a lowest order solution to the Fredholm equation.

\section{Recovery of the approximate analytical solution}

We will now see how to recover the analytic result of equation (47) from the lowest order approximate solution of the Fredholm equation (67). A possible strategy to solve the Fredholm equation is to employ an iterative method. Within this solution scheme, the zeroth order solution is given by [see equation (67)]

$$
r_{k}^{(0)}=-\frac{z_{1}(k)}{v(k)}
$$

Now we notice that for $\varepsilon_{1} \simeq \varepsilon_{2}$ and $E_{F}^{<} \simeq E_{F}^{>}$, the quantity $v(k)$ can be approximated as (see Appendix C)

$$
v(k) \simeq-\frac{2}{\left\langle h_{k}^{>}, e_{0}^{<}\right\rangle+\left\langle e_{k}^{>}, h_{0}^{<}\right\rangle} .
$$

Therefore, we can write the reflection coefficient as

$$
r_{k}^{(0)} \simeq \frac{1}{2} z_{1}(k)\left(\left\langle h_{k}^{>}, e_{0}^{<}\right\rangle+\left\langle e_{k}^{>}, h_{0}^{<}\right\rangle\right) .
$$

Using equation (64) for $z_{1}(k)$, we recover equation (47), that is, the analytical solution as the zeroth order term of the Fredholm equation:

$$
r_{k}^{(0)} \simeq \frac{\left\langle e_{0}^{>}, h_{0}^{<}\right\rangle\left\langle h_{k}^{>}, e_{0}^{<}\right\rangle-\left\langle h_{0}^{>}, e_{0}^{<}\right\rangle\left\langle e_{k}^{>}, h_{0}^{<}\right\rangle}{\left\langle h_{0}^{>}, e_{0}^{<}\right\rangle+\left\langle e_{0}^{>}, h_{0}^{<}\right\rangle} .
$$

We have verified numerically that the approximation given by equation (74) holds with great accuracy even if the conditions for its derivation are violated. This explains the good results given by the analytic approximated solution, even for relatively large contrast between the dielectric constants and the Fermi energies.

\section{RESULTS AND DISCUSSION}

We show the reflection and transmission coefficients for the SPP, $r_{0}$ and $\tau_{0}$, as a function of the plasmon frequency, computed both with the analytic approximation [(43) and (44)] and with the numerical solution of the Fredholm equation (67)
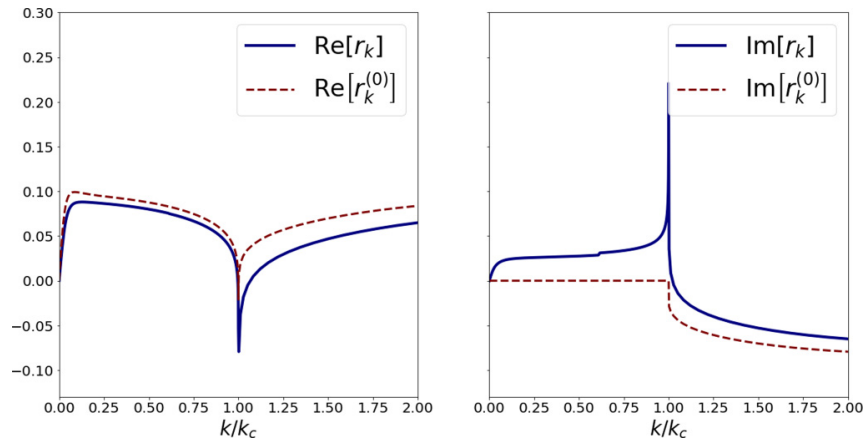

FIG. 5. Real (left panel) and imaginary (right panels) parts of the reflection coefficient $r_{k}$ (divided by $\sqrt{d}$ ) obtained from the numerical solution of the Fredholm equation (solid blue line) as a function of $k / k_{c}$ over the interval $k \in\left[0,2 k_{c}\right]$. The lowest order approximation to the reflection coefficient $r_{k}^{0}$ (73) is also shown by the dashed purple line. The parameters are: $\omega=15.6 \mathrm{meV}, d=300 \mathrm{~nm}, \epsilon_{1}=1.5, \epsilon_{2}=$ $2.5, \epsilon_{3}=4, E_{F}^{<}=0.37 \mathrm{eV}, E_{F}^{>}=0.47 \mathrm{eV}$.

in the right panel of Fig. 4. As can be seen there, the difference between both results is very small, not exceeding $1 \%$. Notice however, that the approximated results overestimate the transmittance of the SPP, which is nevertheless very close to 1. This implies that very little energy is either reflected as a SPP or lost due to emission of radiation. This last statement is further confirmed by the smallness of the reflection and transmission coefficients for radiation modes which are shown as a function of $k / k_{c}$ (with $k_{c}=\sqrt{\epsilon_{3}} k_{0}$ ) in the left panel of Fig. 4. Notice that the reflectance $\left|r_{k}\right|^{2}$ displays a significant dome for $k / k_{c}>1$, highlighting the importance of radiation modes evanescent along the $z$ direction in the field matching at the interface at $z=0$. In Fig. 5, we show the real and imaginary parts of the reflection coefficients $r_{k}$ obtained from the numerical solution of the Fredholm equation and compare it to the lowest order solution as a function of $k / k_{c}$. The agreement is reasonable for the real part, indicating that the approximate analytic expressions indeed provide good results. However, in the imaginary part of the reflection coefficients there is a significant discrepancy close to $k=k_{c}$, with the numerical result displaying there a peak that is absent in the approximate result.

The validity of both the analytic results and the numerical solution can be accessed by studying the total scattered, including the energy carried by the transmitted and reflected SPP and the energy radiated in the scattering process. As a matter of fact, energy conservation implies that $S=1$ (see Appendix B), where

$$
S=\left|r_{0}\right|^{2}+\left|\tau_{0}\right|^{2}+\mathcal{R}_{R}+\mathcal{T}_{R},
$$

with

$$
\begin{aligned}
& \mathcal{R}_{R}=\int_{0}^{k_{c}}\left|r_{k}\right|^{2} d k, \\
& \mathcal{T}_{R}=\int_{0}^{k_{c}}\left|\tau_{k}\right|^{2} d k,
\end{aligned}
$$

respectively, the fraction of energy radiated in reflection and transmission. Notice that the integration only goes up to $k_{c}$, since modes with $k>k_{c}$ are evanescent along the $z$ direction, 

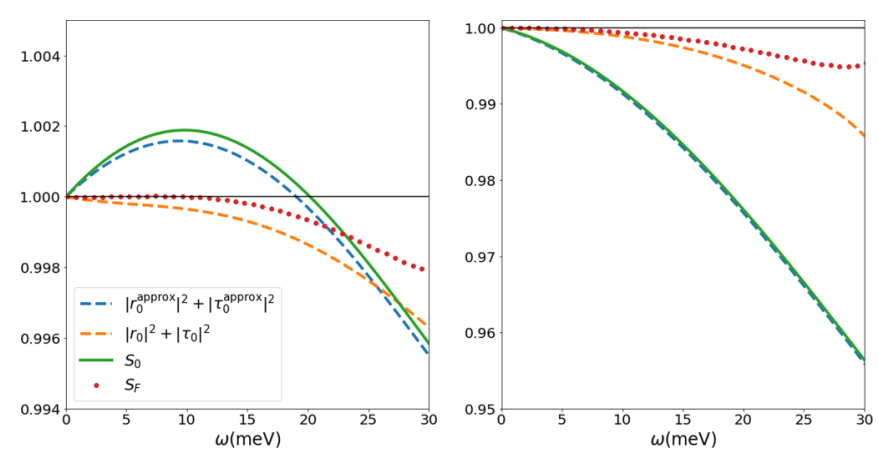

FIG. 6. Sum rule in a large frequency window. The dashed lines refer to $\left|r_{0}\right|^{2}+\left|\tau_{0}\right|^{2}$ for the approximation (blue) and the numerical solution (orange). The green solid line refers to the approximated sum rule $S_{0}$, while the red dotted line refers to the numerical solution $S_{F}$. The parameters used are: $d=300 \mathrm{~nm}, \epsilon_{1}=1.5, \epsilon_{2}=2.5, \epsilon_{3}=$ $4, E_{F}^{<}=0.37 \mathrm{eV}, E_{F}^{>}=0.47 \mathrm{eV}$. The right panel depicts the same quantities but for $E_{F}^{<}=0.3 \mathrm{eV}, E_{F}^{>}=0.6 \mathrm{eV}$. The radiative correction is the difference between the orange dashed line and the red dotted one. We can see the increasing of radiative emission for larger frequencies and higher Fermi energy mismatch.

not carrying energy away for $z \rightarrow \pm \infty$. The statement $S=1$ simply means that the energy of the incident SPP is redistributed into the reflected and transmitted SPP modes and into radiation modes.

Notice that the approximate analytic results in the limits of $\omega / c \rightarrow 0$ and $d \rightarrow 0,(57)$ and (58), imply that $\left|r_{0}\right|^{2}+\left|\tau_{0}\right|^{2}=$ 1. This means that in this limit all the energy is carried by the transmitted and reflected SPP, with no radiation emission. This is expected as in the electrostatic limit no radiation can be emitted. However, in the limit of $\omega / c \rightarrow 0$ and $d \rightarrow \infty$, equations (59) and (60), imply that

$$
\left|r_{0}\right|^{2}+\left|\tau_{0}\right|^{2}=1-\tau_{0} r_{0}^{2} .
$$

Therefore, there is a deviation from the ideal case, $\left|r_{0}\right|^{2}+$ $\left|\tau_{0}\right|^{2}=1$. However, this deviation is small as long as $r_{0} \ll 1$ $\left(\tau_{0} \lesssim 1\right)$. We must point out, however, that the term $\tau_{0} r_{0}^{2}$ cannot be identified with energy losses due to the emission of free radiation, since in the electrostatic limit $(\omega / c \rightarrow 0)$ the propagation of free radiation is forbidden. This deviation is therefore attributed to a limitation of the approximate analytical result.

To check the conservation of energy as a function of frequency, of both the approximate analytic and in the numerical results, we plot in Fig. 6 the energy sum $S$ as a function of the incident plasmon frequency in a range spanning $7.25 \mathrm{THz}$. We see that the analytical results can violate the energy sum rule, leading to $S>1$. The analytical result can also lead to $\left|r_{0}^{\text {approx }}\right|^{2}+\left|\tau_{0}^{\text {approx }}\right|^{2}>1$, which is clearly unphysical, as it would correspond to a generation of energy. This indicates a limitation of the analytic approximation which has also been reported in the scattering of surface phonon polaritons at the interface between two dielectrics [26]. Notice, however, that the violation of the sum rule is actually very small, never exceeding $0.25 \%$ (for $E_{F}^{<}=0.37 \mathrm{eV}$ and $E_{F}^{>}=0.47 \mathrm{eV}$ ). The numerical solution of the Fredholm equation corrects the unphysical result, and we recover $\left|r_{0}^{\text {approx }}\right|^{2}+\left|\tau_{0}^{\text {approx }}\right|^{2} \leq 1$.

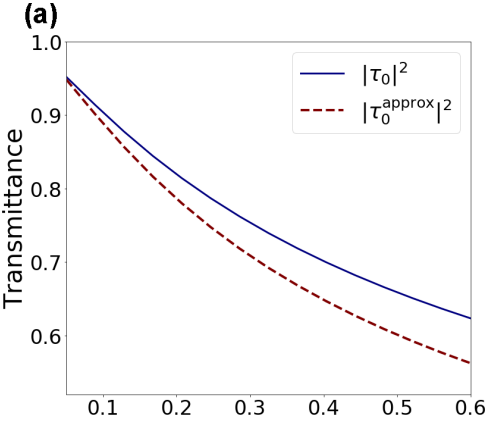

(b)

(c)
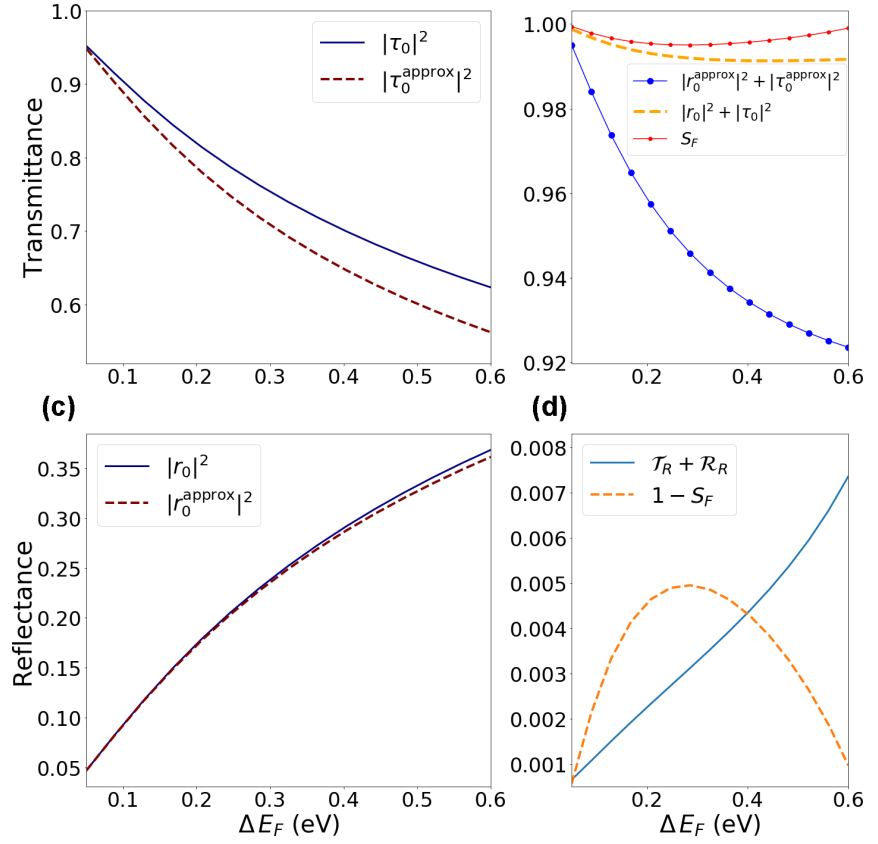

FIG. 7. Dependence of the reflection and transmission coefficients on the graphene Fermi energy contrast $\Delta E_{F}=E_{F}^{>}-E_{F}^{<}$. We fixed $E_{F}^{<}=0.1$ and vary $E_{F}^{>}$from 0.15 to $0.7 \mathrm{eV}$. In panel A (B) we show the transmission (reflection) coefficient for both the analytical (blue solid) and the numerical (red dashed) solutions. In panel B we show the sum $\left|r_{0}\right|^{2}+\left|\tau_{0}\right|^{2}$ also for the analytical (blue circles) and the numerical (dashed orange) solution and the total sum rule, including the radiative modes (red circles). In panel $\mathrm{D}$, we show the energy fraction carried by radiative modes $\mathcal{R}_{R}+\mathcal{T}_{R}$ and the error in the sum rule $1-S_{F}$, with $S_{F}$ calculated using Eq. (77) with the numerical approach. The remaining parameters used in all panels are: $d=300$ $\mathrm{nm}, \epsilon_{1}=1.5, \epsilon_{2}=2.5$, and $\epsilon_{3}=4$.

There is still a small violation of the sum rule which now lies below 1, due to errors induced by the discretization of the integral in the Fredholm equation (67). However, the numerical solution significantly improves the sum rule with the error being less than $0.02 \%$ (for $E_{F}^{<}=0.37 \mathrm{eV}$ and $E_{F}^{>}=0.47 \mathrm{eV}$ ). Notice that as we go to $\omega \rightarrow 0$ the sum rule, in both the approximate analytic (which completely neglects radiation modes) and in the full numerical solutions (where the contribution from the radiative modes is still subjected to errors due to the discretization of the integral), is satisfied to a better degree. This is due to the fact that in the electrostatic limit the contribution due to radiative modes becomes less important. The errors in both methods increase when the graphene conductivity contrast is larger as can be seen in the right panel of Fig. 6 (results obtained for $E_{F}^{<}=0.3 \mathrm{eV}$ and $E_{F}^{>}=0.6 \mathrm{eV}$ ). Since the sum rule is not exactly one, the fraction of energy emitted as radiation can be obtained from $\mathcal{R}+\mathcal{T}=S-\left|r_{0}\right|^{2}-\left|\tau_{0}\right|^{2}$, and can be seen to be extremely small, but increases as the energy of the incident SPP increases and the graphene conductivity contrast is larger.

We further explore the dependence of the scattering on the graphene conductivity contrast in Fig. 7. There we show results, both analytical and numerical, for the dependence of the transmission and reflection coefficients on the graphene Fermi energy contrast between the two regions, $\Delta E_{F}=E_{F}^{>}-E_{F}^{<}$, 
by fixing $E_{F}^{<}=0.1 \mathrm{eV}$ and changing $E_{F}^{>}$from 0.15 to $0.7 \mathrm{eV}$. As expected, by increasing the Fermi energy contrast, the transmission reduces (panel A), while the reflectance increases (panel B), ranging from 10 to $30 \%$. We also point out that the deviation between the analytic approximate result and the numerical one is bigger for the SPP transmission than for the reflectance. We also see that as contrast increases, the analytic approximation becomes less reliable. In panel $\mathrm{C}$ we show the sum rule $\left|r_{0}\right|^{2}+\left|\tau_{0}\right|^{2}$ for the plasmonic modes both for analytical solution and the numerical, and the sum rule $S_{F}$ taking into account the radiative modes. Finally in panel $\mathrm{D}$ we show the error in the sum rule, $1-S_{F}$, and the fraction of energy carried by radiative modes $\mathcal{R}_{R}+\mathcal{T}_{R}$ [see Eqs. (78) and (79)]. The total error never surpasses $0.5 \%$ and the radiative contributions $0.8 \%$ for the parameters considered. Once again, we stress the importance of the inclusion of the radiative modes in the calculation of the plasmonic and transmission coefficients.

\section{CONCLUSIONS}

We have analyzed in detail the scattering of graphene surface plasmon polaritons at a sharp graphene conductivity step and/or change of the dielectric substrate. One of the merits of our calculation is the ability to provide analytic expressions for the reflectance and transmittance amplitudes for arbitrary values of the graphene sheet conductivity and of the surrounding dielectric constants, in a realistic geometric configuration. Although the analytical approach is not exact, it is good enough to estimate the values of $r_{0}$ and $\tau_{0}$, which can be corrected either by an iterative solution or a fully numerical solution (see Appendix D) of the Fredholm equation. The corrections are, however, small. The calculation also predicts that the emission of free radiation in the scattering event is small. This situation is rather favorable for plasmon scattering, as most of the energy remains in the plasmon field and is not lost to the radiation continuum.

Note that our calculations are realistic in what concerns the geometry of the system, since the metallic gate is taken into account as is the existence of two different dielectrics underneath graphene. However, we assumed that the induced change of the graphene conductivity is abrupt at the interface. A more realistic situation would be to consider a smooth transition of the electronic density across the interface. In this case, the reflection coefficients are no longer well defined, except faraway from the region where the conductivity changes; this renders the calculation much more difficult. Nevertheless, our results should remain valid provided the incident plasmon wavelength is much larger than the length scale over which the graphene conductivity changes.

The method employed in this paper can be extended to take into account the coupling of the SPP to the substrate's surface optical phonons, as for example in $\mathrm{SiO}_{2}$, by taking into account the frequency dependence of the dielectric function of the substrate. It is also possible to generalize the present method to a geometry where a finite dielectric is sandwiched between two semi-infinite ones. In this setup, by adjusting the length of the central dielectric it is possible to achieve either total transmission or total reflection via Fabry-Pérot oscillations, thus allowing the construction of a Bragg reflector. Alternatively, we can change the value of the gate potential, thus tuning the frequency for which there is total reflection or total transmittance. This give us a real time and on-demand control on the scattering of the plasmon. We point out that we have only focused on the case of scattering at normal incidence. However, the method of eigenmode field expansion and matching employed in this work can also be generalized and applied to the case of oblique incidence. That extension will be the goal of a forthcoming publication.

\section{ACKNOWLEDGMENTS}

A.J.C. acknowledges the scholarship from the Brazilian agency $\mathrm{CNPq}$ (Conselho Nacional de Desenvolvimento Científico e Tecnológico). B.A., Y.V.B., and N.M.R.P. acknowledge support from the European Commission through the project "Graphene-Driven Revolutions in ICT and Beyond" (Ref. No. 696656). P.A.D.G. acknowledges financial support from the VILLUM Fonden (Grant No. 16498), and from the Center for Nanostructured Graphene sponsored by the Danish National Research Foundation (project DNRF103). N.M.R.P. also acknowledges the hospitality of the MackGraphe Center, at Mackenzie Presbyterian University, where this work has started, the projects Fapesp 2012/50259-8 and 2016/11814-7, and the Portuguese Foundation for Science and Technology (FCT) in the framework of the Strategic Financing UID/FIS/04650/2013.

\section{APPENDIX A: EIGENMODES OF MAXWELL'S EQUATIONS}

In this Appendix we determine the eigenmodes of the system represented in Fig. 1 for each to the regions $z \lessgtr 0$, by solving Maxwell's equations in this geometry. The electric $\mathbf{E}$ and the magnetic $\mathbf{B}$ fields are governed by the inhomogeneous Maxwell equations

$$
\begin{gathered}
\nabla \times \mathbf{E}=-\frac{\partial \mathbf{B}}{\partial t}, \\
\nabla \times \mathbf{B}=\frac{\varepsilon(x, z)}{c^{2}} \frac{\partial \mathbf{E}}{\partial t}+\mu_{0} \mathbf{j},
\end{gathered}
$$

where $\mathbf{j}$ is the current density due to the graphene layer at $x=0$ and $\epsilon(x, z)$ takes into account the inhomogeneous dielectric environment that surrounds the graphene layer. $\epsilon(x, z)$ is piecewise homogeneous and we write it as $\epsilon(x, z)=\epsilon^{<}(x)$ for $z<0$ and $\epsilon(x, z)=\epsilon^{>}(x)$ for $z>0$, with

$$
\begin{aligned}
& \epsilon^{<}(x)= \begin{cases}\epsilon_{3}, & x>0 \\
\epsilon_{1}, & 0>x>-d\end{cases} \\
& \epsilon^{>}(x)= \begin{cases}\epsilon_{3}, & x>0 \\
\epsilon_{2}, & 0>x>-d\end{cases}
\end{aligned}
$$

The graphene current density is related to the electric field by $\mathbf{j}=\sigma(z) \mathbf{E}_{\perp}$, where $\mathbf{E}_{\perp}$ represents the components of $\mathbf{E}$ that are perpendicular to the $x$ direction. We also allow for different graphene conductivities (due to different local doping levels) for $z<0$ and $z>0$, respectively, $\sigma_{<}$and $\sigma_{>}$. We will use the Drude model for the graphene conductivity, namely

$$
\sigma_{\lessgtr}=\frac{e^{2}}{\pi \hbar} \frac{E_{F}^{\lessgtr}}{\gamma \lessgtr+i \hbar \omega}
$$

with $E_{F}^{\lessgtr}$ the local Fermi level and $\gamma \lessgtr$ the local decay rate. 
We will consider that all fields have a harmonic time dependence of the form $e^{i \omega t}$ and also assume that the system is translationally invariant along the $y$ direction. We want to describe scattering at normal incident and therefore we can drop all dependence of the problem on the $y$ coordinate (i.e., $\partial / \partial y=0)$. The total electromagnetic field can, in general, be split in two polarizations: $s / \mathrm{TE}$ (transverse electric) polarization and $p /$ TM (transverse magnetic) polarization. Since the SPPs are TM-polarized waves, further in the Appendix we restrict our consideration to that particular polarization. For this polarization and at normal incidence, the electric field will have nonzero $x$ and $z$ components, $\mathbf{E}=\left(E_{x}, 0, E_{z}\right)$, while the only nonzero component of the magnetic field is the $y$ component, $\mathbf{B}=\left(0, B_{y}, 0\right)$. Under these conditions we rewrite Maxwell's equations (A1) and (A2) as

$$
\begin{gathered}
\frac{\partial E_{z}}{\partial x}-\frac{\partial E_{x}}{\partial z}=i \omega B_{y}, \\
-\frac{\partial B_{y}}{\partial z}=i \frac{\omega \varepsilon(x, z)}{c^{2}} E_{x}, \\
\frac{\partial B_{y}}{\partial x}=i \frac{\omega \varepsilon(x, z)}{c^{2}} E_{z}+\mu_{0} \delta(x) \sigma(z) E_{z} .
\end{gathered}
$$

Due to the piecewise homogeneity of the system along the $z$ direction, we can study separately the electromagnetic fields in the regions $z<0$ and $z>0$. In general, there is a series of solutions, which we will refer to as eigenmodes, indexed by some label $n$ for each of the regions $z \lessgtr 0$. A general solution for each region can be represented as a superposition of these eigenmodes. In particular, the expression for the $y$ component of the magnetic field at $z \lessgtr 0$ have the form

$$
B_{y}^{\lessgtr}(x, z)=\sum_{n} \alpha_{n, \lambda} e^{\lambda i q_{n}^{\lessgtr} z} h_{n}^{\lessgtr}(x),
$$

while the nonzero components of the electric field are

$$
\begin{gathered}
E_{x}^{\lessgtr}(x, z)=-\sum_{n,} \pm \alpha_{n, \lambda} e^{\lambda i q_{n}^{\lessgtr} z} e_{n}^{\lessgtr}(x), \\
E_{z}^{\lessgtr}(x, z)=\sum_{n} \alpha_{n, \lambda} e^{\lambda i q_{n}} z \mathcal{E}_{n}^{\lessgtr}(x) .
\end{gathered}
$$

$\alpha_{n, \lambda}$ are the eigenmode amplitudes and the summation is taken with respect to the eigenmode index $n$. The $\lambda= \pm 1$ sign stands for the left/right propagating waves in the $z$ direction with wave number $q_{n}^{\lessgtr}$. With some abuse of notation, the summation symbol in equations (A9)-(A11) actually represents a summation, an integral or both, depending if the basis is discrete and/or continuous.

From equations (A6)-(A8), for each mode the functions $h_{n}^{\lessgtr}, e_{n}^{\lessgtr}$, and $\mathcal{E}_{n}^{\lessgtr}$ are solutions of the equations

$$
\begin{gathered}
\frac{\partial \mathcal{E}_{n}^{\lessgtr}}{\partial x}+i q_{n}^{\lessgtr} e_{n}^{\lessgtr}(x)=i \omega h_{n}^{\lessgtr}(x), \\
i q_{n}^{\lessgtr} h_{n}^{\lessgtr}(x)=i \frac{\omega \varepsilon \gtrless(x)}{c^{2}} e_{n}^{\lessgtr}(x), \\
\frac{\partial h_{n}^{\lessgtr}}{\partial x}=\left[i \frac{\omega \varepsilon}{c^{2}}+\mu_{0} \delta(x) \sigma_{\lessgtr}\right] \mathcal{E}_{n}^{\lessgtr}(x) .
\end{gathered}
$$

As before, the piecewise homogeneity of equations (A12) (A14) along the $x$ direction allows us to solve them separately in regions $-d<x<0$ and $x>0$ and then apply the boundary conditions. Thus, in the region $x>0$, occupied by the dielectric $\epsilon_{3}$ substitution of equations (A13) and (A14) into equation (A12) results in the wave equation

$$
\frac{d^{2} h_{n}^{\lessgtr}(x)}{d x^{2}}=(p \underset{3 \| n}{\lessgtr})^{2} h_{n}^{\gtrless}(x),
$$

In the same way, for region $-d<x<0$, we obtain the wave equations

$$
\begin{aligned}
& \frac{d^{2} h_{n}^{<}(x)}{d x^{2}}=\left(p_{1 \mid n}\right)^{2} h_{n}^{<}(x), \\
& \frac{d^{2} h_{n}^{>}(x)}{d x^{2}}=\left(p_{2 \mid n}\right)^{2} h_{n}^{>}(x),
\end{aligned}
$$

which are valid for the domains $z<0$ and $z>0$, respectively. In equations (A15)-(A17) $\left(p_{1 \mid n}\right)^{2}=\left(q_{n}^{<}\right)^{2}-\epsilon_{1} k_{0}^{2},\left(p_{2 \mid n}^{>}\right)^{2}=$ $\left(q_{n}^{>}\right)^{2}-\epsilon_{2} k_{0}^{2}$, and $\left(p_{3 \mid n}^{\lessgtr}\right)^{2}=\left(q_{n}^{\lessgtr}\right)^{2}-\epsilon_{3} k_{0}^{2}$, with $k_{0}=\omega / c$ the wave number in vacuum. Notice that $q_{n}^{\lessgtr}$ is the same in both $x>0$ and $0>x>-d$ regions. The fact that we have a perfect metal at $x=-d$ forces the $z$ component of the electric field to become null there. Therefore, the magnetic field mode along the $y$ component must have the following form

$$
h_{n}^{\lessgtr}(x)=\left\{\begin{array}{ll}
B_{n}^{\lessgtr} e^{p_{3 \mid n} x}+C_{n}^{\lessgtr} e^{-p_{3 \mid n} x}, & x>0 \\
A_{n}^{\lessgtr} \cosh \left[p_{j \mid n}(x+d)\right], & 0>x>-d
\end{array},\right.
$$

with the $x$ component of the electric field given by

$$
e_{n}^{\lessgtr}(x)=\frac{q_{n}^{\lessgtr} c^{2}}{\omega \epsilon_{3}}\left\{\begin{array}{ll}
B_{n}^{\lessgtr} e^{p_{3 \mid n} x}+C_{n}^{\lessgtr} e^{-p_{3 \mid n} x}, & x>0 \\
\frac{\epsilon_{3}}{\epsilon_{j}} A_{n}^{\lessgtr} \cosh \left[p_{j \mid n}(x+d)\right], & 0>x>-d
\end{array},\right.
$$

and the $z$ component being given by

$$
\begin{aligned}
& \mathcal{E}_{n}^{\lessgtr}(x) \\
& =\frac{p_{3 \mid n}^{\lessgtr} c^{2}}{i \omega \epsilon_{3}}\left\{\begin{array}{ll}
B_{n}^{\lessgtr} e^{p_{3 \mid n} x}-C_{n}^{\lessgtr} e^{-p_{3 \mid n} x}, & x>0 \\
\frac{p_{j \mid n} \epsilon_{3}}{p_{3 \mid n}^{\lessgtr} \epsilon_{j}} A_{n}^{\lessgtr} \sinh \left[p_{j \mid n}(x+d)\right], & 0>x>-d
\end{array} .\right.
\end{aligned}
$$

Notice that in equations (A18)-(A20) the subscript $j=2$ is for $z>0$ (and is combined with the superscript $>$ ), while the subscript $j=1$ is for $z<0$ (combined with the superscript $<$ ). Also $A_{n}^{\lessgtr}, B_{n}^{\lessgtr}$, and $C_{n}^{\lessgtr}$ are coefficients to be determined such that boundary conditions at $x=0$ are satisfied and the mode is normalized. Integration of equations (A12)-(A14) in the limits from $x=0^{-}$to $x=0^{+}$imposes the following boundary conditions at $x=0$

$$
\begin{gathered}
\mathcal{E}_{n}^{\lessgtr}\left(0^{+}\right)-\mathcal{E}_{n}^{\lessgtr}\left(0^{-}\right)=0, \\
b_{n}^{\lessgtr}\left(0^{+}\right)-b_{n}^{\lessgtr}\left(0^{-}\right)=\mu_{0} \sigma_{\lessgtr} \mathcal{E}_{n}^{\lessgtr}(0),
\end{gathered}
$$


which translate into the following equations for $A_{n}^{\lessgtr}, B_{n}^{\lessgtr}$, and $C_{n}^{\lessgtr}$

$$
\begin{gathered}
B_{n}^{\lessgtr}-C_{n}^{\lessgtr}=\frac{p_{j \mid n} \epsilon_{3}}{p_{3 \mid n}^{\lessgtr} \epsilon_{j}} A_{n}^{\lessgtr} \sinh \left(p_{j \mid n} d\right), \\
B_{n}^{\lessgtr}+C_{n}^{\lessgtr}-A_{n}^{\lessgtr} \cosh \left(p_{j \mid n} d\right)=\frac{\sigma_{\lessgtr}}{i \omega \epsilon_{0}} \frac{p_{j \mid n}}{\epsilon_{j}} A^{\lessgtr} \sinh \left(p_{j \mid n} d\right) .
\end{gathered}
$$

By solving these equations for $B_{n}^{\lessgtr}$ and $C_{n}^{\lessgtr}$ we obtain equations (21) and (22) of the main text.

The normalization condition (27) allows us to fix the value of $A_{n}^{\lessgtr}$. By using the following results

$$
\begin{aligned}
& \frac{2}{\pi} \int_{0}^{+\infty} d x \cos (k x) \cos \left(k^{\prime} x\right)=\delta\left(k-k^{\prime}\right), \\
& \frac{2}{\pi} \int_{0}^{+\infty} d x \sin (k x) \sin \left(k^{\prime} x\right)=\delta\left(k-k^{\prime}\right), \\
& \int_{0}^{+\infty} d x \cos (k x) \sin \left(k^{\prime} x\right)=\frac{k^{\prime}}{\left(k^{\prime}\right)^{2}-k^{2}},
\end{aligned}
$$

we obtain equation (28) of the main text.

\section{APPENDIX B: ENERGY SUM RULE}

Energy propagation is intimately related to the time average of the Poynting vector $\mathbf{S}$, defined as

$$
\mathbf{S}=\frac{1}{2 \mu_{0}} \mathbf{E} \times \mathbf{B}^{*}
$$

For a TM-polarized electromagnetic field propagating along the $z$ direction, the Poynting vector has the explicit form

$$
\mathbf{S}=\frac{1}{2 \mu_{0}}\left(E_{x} B_{y}^{*} \mathbf{u}_{z}-E_{z} B_{y}^{*} \mathbf{u}_{x}\right) .
$$

In the presence of an imaginary-only conductivity energy is conserved and Poynting's theorem establishes that

$$
\operatorname{Re} \int_{\partial V} \mathbf{S} \cdot d \mathbf{A}=0
$$

where $\partial V$ is the closed surface enclosing the volume $V$ and $d \mathbf{A}$ is an infinitesimal areal vector lying on the surface of $\partial V$ and pointing from the inside to the outside of the volume $V$. We are interested in the fields in the far field, therefore we draw a cube passing through $z= \pm \infty, x=-d, x=+\infty$, and $y= \pm \infty$. As the fields do not depend on $y$ the integral over $\partial V$ can be reduced to a one-dimensional integral along the rectangle defined by $z= \pm \infty, x=-d$, and $x=+\infty$. We now use equations (29)-(32) to compute the Poynting vector. The energy flow along the $z$ direction is related to $2 \mu_{0} S_{z}^{<}(x, z)$ which reads

$$
\begin{aligned}
& 2 \mu_{0} S_{z}^{<}(x, z) \\
& =E_{x}^{<}(x, z) B_{y}^{<*}(x, z) \\
& =e_{0}^{<}(x)\left(e^{-i q_{0}^{<} z}-r_{0} e^{i q_{0}^{<} z}\right) h_{0}^{<*}(x)\left(e^{i q_{0}^{<} z}+r_{0}^{*} e^{-i q_{0}^{<} z}\right) \\
& \quad-\int_{0}^{\infty} d k \int_{0}^{\infty} d k^{\prime} r_{k} r_{k^{\prime}}^{*} e_{k}^{<}(x) h_{k^{\prime}}^{<*}(x) e^{i\left(q_{k}^{<}-q_{k^{\prime}}^{<*}\right) z}
\end{aligned}
$$

$$
\begin{aligned}
& -\int_{0}^{\infty} d k r_{k} e_{k}^{<}(x) e^{i q_{k}^{<} z} h_{0}^{<*}(x)\left(e^{i q_{0}^{<} z}+r_{0}^{*} e^{-i q_{0}^{<} z}\right) \\
& +\int_{0}^{\infty} d k r_{k}^{*} h_{k}^{<*}(x) e^{-i q_{k}^{<*} z} e_{0}^{<}(x)\left(e^{-i q_{0}^{<} z}-r_{0} e^{i q_{0}^{<} z}\right) .
\end{aligned}
$$

Integrating $2 \mu_{0} S_{z}^{<}(x, z)$ along the $x$ axis from $x=-d$ to $x=$ $\infty$ and using the orthonormality of the modes it follows that

$$
\begin{aligned}
& 2 \mu_{0} \operatorname{Re} \int_{-d}^{\infty} d x\left[S_{z}^{<}(x, z \rightarrow-\infty)\right] \\
& \quad=\left(1-\left|r_{0}\right|^{2}\right)-\int_{0}^{k_{c}} d k\left|r_{k}\right|^{2}-\int_{k_{c}}^{\infty} d k\left|r_{k}\right|^{2} e^{2\left|q_{R}^{<}\right| z} .
\end{aligned}
$$

In the far field $z \rightarrow-\infty$ the last term of the previous equation is zero. In the same way the contribution from the surface located at $z=+\infty$ provides the result:

$$
2 \mu_{0} \operatorname{Re} \int_{-d}^{\infty} d x S_{z}^{>}(x, z \rightarrow \infty)=\left|\tau_{0}\right|^{2}+\int_{0}^{k_{c}} d k\left|\tau_{k}\right|^{2} .
$$

Finally, we still need the contribution from the line at $x=+\infty$. The last term we need to compute is:

$$
\begin{aligned}
& \operatorname{Re} \int_{-\infty}^{\infty} d z S_{x}(x, z) \\
& =\operatorname{Re} \int_{-\infty}^{0} d z S_{x}^{<}(x, z)+\operatorname{Re} \int_{0}^{\infty} d z S_{x}^{>}(x, z),
\end{aligned}
$$

which corresponds to radiation emitted orthogonal to the graphene plane. The plasmonic fields $e_{0}$ and $h_{0}$ go to zero when $x \rightarrow \infty$ and thus do not transport energy. Therefore we are left with the term that depends on the radiative modes. It can be shown that the integral is purely imaginary and therefore its real part is zero and does not contribute to energy conservation. Putting all together in equation (B3) we find

$$
1=\left|\tau_{0}\right|^{2}+\left|r_{0}\right|^{2}+\int_{0}^{k_{c}} d k\left|r_{k}\right|^{2}+\int_{0}^{k_{c}} d k\left|\tau_{k}\right|^{2},
$$

which is the statement of energy conservation.

\section{APPENDIX C: EXPLICIT FORM OF THE INNER PRODUCTS}

In this Appendix we list the explicit results for the inner products. First we provide results for some useful integrals:

$$
\begin{gathered}
\int_{-d}^{0} d x \cosh \left(p_{j}(x+d)\right) \cosh \left(p_{j^{\prime}}(x+d)\right) \\
=\frac{1}{2}\left[\frac{\sinh \left(p_{j}+p_{j^{\prime}}\right) d}{p_{j}+p_{j}^{\prime}}+\frac{\sinh \left[\left(p_{j}-p_{j}^{\prime}\right) d\right]}{p_{j}-p_{j^{\prime}}}\right], \\
\int_{0}^{\infty} d x e^{-2 p_{3} x}=\frac{1}{2 p_{3}}, \\
\int_{0}^{\infty} d x e^{-p_{3} x} \sin (k x)=\frac{k}{k^{2}+p_{3}^{2}}, \\
\int_{0}^{\infty} d x e^{-p_{3} x} \cos (k x)=\frac{p_{3}}{k^{2}+p_{3}^{2}},
\end{gathered}
$$


and

$$
\int_{0}^{\infty} d x \cos (k x) \sin \left(k^{\prime} x\right) e^{-0^{+} x}=\frac{1}{2}\left(\frac{1}{k+k^{\prime}}+\frac{1}{k^{\prime}-k}\right) .
$$

Using the previous integrals we can compute the different inner products, which, after tedious calculations, read:

$$
\begin{aligned}
& \left\langle e_{0}^{<}, h_{0}^{>}\right\rangle=q^{<} \tilde{A}_{1} \tilde{A}_{2}\left[\frac{1}{\varepsilon_{1}} S\left(p_{1}, p_{2}\right)+\frac{\varepsilon_{3}}{\tilde{p}_{3}^{<}+\tilde{p}_{3}^{>}} a_{1} a_{2}\right], \\
& \left\langle h_{0}^{<}, e_{0}^{>}\right\rangle=q^{>} \tilde{A}_{1} \tilde{A}_{2}\left[\frac{1}{\varepsilon_{2}} S\left(p_{1}, p_{2}\right)+\frac{\varepsilon_{3}}{\tilde{p}_{3}^{<}+\tilde{p}_{3}^{>}} a_{1} a_{2}\right] \text {, } \\
& \left\langle e^{<}(k), h_{0}^{>}\right\rangle \\
& =\sqrt{d} \tilde{q}^{R} \tilde{A}_{1}^{R}(\tilde{k}) \tilde{A}_{2}\left(\frac{1}{\varepsilon_{1}} S\left(\tilde{p}_{1}^{R}, \tilde{p}_{2}\right)+\frac{a_{1}}{\tilde{k}^{2}+\tilde{p}_{3}^{>2}} T_{1}(\tilde{k})\right), \\
& \left\langle h^{<}(k), e_{0}^{>}\right\rangle=\sqrt{d} \tilde{q}^{>} \tilde{A}_{1}^{R}(\tilde{k}) \tilde{A}_{2}\left(\frac{1}{\varepsilon_{2}} S\left(\tilde{p}_{1}^{R}, \tilde{p}_{2}\right)\right. \\
& \left.+\frac{a_{1}}{\tilde{k}^{2}+\tilde{p}_{3}^{>2}} T_{1}(\tilde{k})\right) \text {, } \\
& \left\langle e_{0}^{<}, h^{>}(k)\right\rangle=\sqrt{d} \tilde{q}^{<} \tilde{A}_{1} \tilde{A}_{2}^{R}(\tilde{k})\left(\frac{1}{\varepsilon_{1}} S\left(\tilde{p}_{1}, \tilde{p}_{2}^{R}\right)\right. \\
& \left.+\frac{a_{2}}{\tilde{k}^{2}+\tilde{p}_{3}^{<2}} T_{1}(\tilde{k})\right) \text {, } \\
& \left\langle h_{0}^{<}, e^{>}(k)\right\rangle=\sqrt{d} \tilde{q}^{R} \tilde{A}_{1} \tilde{A}_{2}^{R}(\tilde{k})\left(\frac{1}{\varepsilon_{2}} S\left(\tilde{p}_{1}, \tilde{p}_{2}^{R}\right)\right. \\
& \left.+\frac{a_{2}}{\tilde{k}^{2}+\tilde{p}_{3}^{<2}} T_{1}(\tilde{k})\right) \text {, } \\
& \left\langle e^{<}\left(k^{\prime}\right), h^{>}(k)\right\rangle=\sqrt{d} \tilde{q}^{R}\left(k^{\prime}\right) A_{1}^{R}\left(k^{\prime}\right) A_{2}^{R}(k)\left[\frac{1}{\varepsilon_{1}} S\left(\tilde{p}_{1}^{R \prime}, \tilde{p}_{2}^{R}\right)+\right. \\
& \left.+\frac{2}{\pi \varepsilon_{3}} Q_{1}(k) \delta\left(k-k^{\prime}\right)+\frac{Q_{2}\left(k, k^{\prime}\right)}{k^{2}-k^{\prime 2}}\right] \text {, }
\end{aligned}
$$

$$
\begin{aligned}
\left\langle h^{<}\left(k^{\prime}\right), e^{>}(k)\right\rangle= & \sqrt{d} \tilde{q}^{R}(k) A_{1}^{R}\left(k^{\prime}\right) A_{2}^{R}(k)\left[\frac{1}{\varepsilon_{2}} S\left(\tilde{p}_{1}^{R^{\prime}}, \tilde{p}_{2}^{R}\right)+\right. \\
& \left.+\frac{2}{\pi \varepsilon_{3}} Q_{1}(k) \delta\left(\tilde{k}-\tilde{k}^{\prime}\right)+\frac{Q_{2}\left(k, k^{\prime}\right)}{\tilde{k}^{2}-\left(\tilde{k}^{\prime}\right)^{2}}\right],
\end{aligned}
$$

where we have defined the functions

$$
\begin{gathered}
S(x, y)=\frac{1}{2}\left(\frac{\sinh (x+y)}{x+y}+\frac{\sinh (x-y)}{x-y}\right), \\
a_{i}=\frac{\cosh \left(\tilde{p}_{3}^{i}\right)}{\varepsilon_{3}}\left(1-\frac{\tilde{p}_{3}^{i} \sigma_{i}^{I}}{\omega d \varepsilon_{0} \varepsilon_{3}}\right)^{-1},
\end{gathered}
$$

where $\tilde{p}_{3}^{1}=p_{3}^{<} d, \tilde{p}_{3}^{2}=p_{3}^{>} d, \tilde{k}=k d$, and:

$$
\begin{gathered}
T_{i}(k)=R_{i}(k) \tilde{p}_{3}^{i}+Z_{i}(k), \\
R_{i}(k)=\cosh \left(\tilde{p}_{i}^{R}\right)-\frac{\sigma_{i}^{I} \tilde{p}_{i}^{R}}{\omega d \varepsilon_{i} \varepsilon_{0}} \sinh \left(\tilde{p}_{i}^{R}\right), \\
Z_{i}(k)=\frac{\tilde{p}_{i}^{R}}{\varepsilon_{i}} \sinh \left(\tilde{p}_{i}^{R}\right), \\
Q_{1}(k)=R_{1}(\tilde{k}) R_{2}(\tilde{k})+\frac{1}{\tilde{k}^{2}} Z_{1}(\tilde{k}) Z_{2}(\tilde{k}), \\
Q_{2}\left(k, k^{\prime}\right)=-R_{1}\left(k^{\prime}\right) \tilde{p}_{2}^{R}(k)+R_{2}(k) \tilde{p}_{1}^{R}\left(k^{\prime}\right), \\
\tilde{q}^{R}(k)=d \sqrt{\epsilon_{3} k_{0}^{2}-k^{2},} \\
\tilde{p}_{i}^{R}=\tilde{p}_{i}^{R}(k)=d \sqrt{\varepsilon_{i} k_{0}^{2}-k^{2}}, \\
\tilde{A}_{i}^{R}(k)=\sqrt{\frac{c^{2}}{\omega}} A_{k}^{\lessgtr}, \\
\tilde{A}_{i}=A_{0}^{\lessgtr},
\end{gathered}
$$

where in the last two equations, $i=1(i=2)$ for the superscript $<(>)$.

From equations (C12) and (C13) and from equation (64) the function $v(k)$, as defined by equation (66), is given by

$$
v(k)=-\frac{4}{\pi \epsilon_{3}} \frac{c^{2}}{\omega} \frac{Q_{1}(k) \tilde{q}^{R}(k) A_{k}^{>} A_{k}^{<}}{\left\langle h_{k}^{>}, e_{0}^{<}\right\rangle+\left\langle e_{k}^{>}, h_{0}^{<}\right\rangle} .
$$

We point out that for $\varepsilon_{1} \simeq \varepsilon_{2}$ and $E_{F}^{<} \simeq E_{F}^{>}$we have that

$$
\frac{4}{\pi \epsilon_{3}} \frac{c^{2}}{\omega} Q_{1}(k) \tilde{q}^{R}(k) A_{k}^{>} A_{k}^{<} \simeq 2,
$$

such that we obtain

$$
v(k) \simeq-\frac{2}{\left\langle h_{k}^{>}, e_{0}^{<}\right\rangle+\left\langle e_{k}^{>}, h_{0}^{<}\right\rangle} .
$$

\section{APPENDIX D: NUMERICAL SOLUTION OF FREDHOLM PROBLEM}

To solve the Fredholm equation (67), first we introduce a cutoff $k_{\max }=c_{F} k_{c}$ in the integral, where $c_{F}$ is large and is chosen as the value needed for the solution to converge. The kernel of the Fredholm equation, $z_{3}\left(k, k^{\prime}\right)$, has a divergence of the kind:

$$
\frac{1}{k-k^{\prime}}
$$

that comes from the term proportional to $Q_{2}\left(k, k^{\prime}\right)$ in the inner products $(\mathrm{C} 12)$ and $(\mathrm{C} 13)$. To regularize this divergence, we make the substitution:

$$
\frac{1}{k-k^{\prime}} \rightarrow \frac{k-k^{\prime}}{\left(k-k^{\prime}\right)^{2}+\eta^{2}},
$$

where $\eta$ is a parameter chosen as small as necessary to achieve convergence of the calculation. In the numerical results shown in the main text, we used $c_{F}=30$ and $\eta=10^{-3} k_{c}$. 
In the integral of equation (67) we make the variable change $u=k_{c} k$ and separate the integration limit in two parts:

$$
\int_{0}^{c_{F}} d u=\int_{0}^{1} d u+\int_{1}^{c_{F}} d u .
$$

Next, we divide each of those integrals in $N_{1}$ and $N_{2}$ equally spaced regions. For each of those regions, we apply a GaussLegendre quadrature with $N_{\text {Gauss } 1}$ (when $u<1$ ) and $N_{\text {Gauss2 }}$ $(u>1)$ points. The Fredholm problem now is transformed into a matrix equation:

$$
\boldsymbol{r}=\boldsymbol{r}_{0}-\boldsymbol{Z}_{3} \cdot \boldsymbol{r}
$$

where $Z_{3}$ is a $\left(N_{1} N_{\text {Gauss } 1}+N_{2} N_{\text {Gauss } 2}\right) \times\left(N_{1} N_{\text {Gauss } 1}+\right.$ $N_{2} N_{\text {Gauss2 }}$ ) matrix obtained from the discretization of the kernel $z_{3}\left(k, k^{\prime}\right), \boldsymbol{r}$ is the solution we seek, being a vector obtained by discretizing the reflection coefficient, and $\boldsymbol{r}_{0}$ is a vector obtained from the discretization of the zeroth order solution of the Fredholm equation (73). The solution of (D4) is obtained trivially as $\boldsymbol{r}=\left(1+Z_{3}\right)^{-1} \cdot \boldsymbol{r}_{0}$. For the results shown in this paper we used $N_{1}=N_{2}=80, N_{\text {Gauss } 1}=2, N_{\text {Gauss2 }}=3$.

This numerical procedure works for the spectral range shown in this paper (frequencies up to $7.25 \mathrm{THz}$ ). For higher frequencies, the integration of the resulting $\tau_{k}$ function, to calculate the sum rule (77), diverges due to the singularity at the $k_{c}$ point (see Fig. 4). To go to higher frequencies, a more sophisticated integration algorithm is necessary.
[1] P. A. D. Gonçalves and N. M. R. Peres, An Introduction to Graphene Plasmonics (World Scientific, Singapore, 2016).

[2] Y. V. Bludov, A. Ferreira, N. M. R. Peres, and M. I. Vasileskiy, Int. J. Mod. Phys. B 27, 1341001 (2013).

[3] S. Xiao, X. Zhu, B.-H. Li, and N. A. Mortensen, Frontiers of Physics 11, 117801 (2016).

[4] R. F. Oulton, D. F. P. Pile, Y. Liu, and X. Zhang, Phys. Rev. B 76, 035408 (2007).

[5] D. Ballester, M. S. Tame, and M. S. Kim, Phys. Rev. A 82, 012325 (2010).

[6] A. H. Castro Neto, F. Guinea, N. M. R. Peres, K. S. Novoselov, and A. K. Geim, Rev. Mod. Phys. 81, 109 (2009).

[7] N. M. R. Peres, Rev. Mod. Phys. 82, 2673 (2010).

[8] L. Lin, L. Liao, J. Yin, H. Peng, and Z. Liu, Nano Today 10, 701 (2015).

[9] J. A. Sánchez-Gil and A. A. Maradudin, Phys. Rev. B 60, 8359 (1999).

[10] J. Polanco, R. M. Fitzgerald, and A. A. Maradudin, Phys. Rev. B 87, 155417 (2013).

[11] B. Rejaei and A. Khavasi, J. Opt. 17, 075002 (2015).

[12] S. Farajollahi, B. Rejaei, and A. Khavasi, J. Opt. 18, 075005 (2016).

[13] J. L. Garcia-Pomar, A. Y. Nikitin, and L. Martin-Moreno, ACS Nano 7, 4988 (2013).

[14] A. Y. Nikitin, T. Low, and L. Martin-Moreno, Phys. Rev. B 90, 041407 (2014).
[15] Z. Fei, A. S. Rodin, W. Gannett, S. Dai, W. Regan, M. Wagner, M. K. Liu, A. S. McLeod, G. Dominguez, M. Thiemens, A. H. Castro Neto, F. Keilmann, A. Zettl, R. Hillenbrand, M. M. Fogler, and D. N. Basov, Nat Nano 8, 821 (2013).

[16] Z. Fei, G.-X. Ni, B.-Y. Jiang, M. M. Fogler, and D. N. Basov, ACS Photonics 4, 2971 (2017).

[17] T. M. Slipchenko, M. L. Nesterov, R. Hillenbrand, A. Y. Nikitin, and L. Martín-Moreno, ACS Photonics 4, 3081 (2017).

[18] I. I. Smolyaninov, D. L. Mazzoni, J. Mait, and C. C. Davis, Phys. Rev. B 56, 1601 (1997).

[19] N. Rotenberg, M. Spasenović, T. L. Krijger, B. le Feber, F. J. García de Abajo, and L. Kuipers, Phys. Rev. Lett. 108, 127402 (2012).

[20] R. Gordon, Phys. Rev. B 74, 153417 (2006).

[21] O. You, B. Bai, X. Wu, Z. Zhu, and Q. Wang, Opt. Lett. 40, 5486 (2015).

[22] B. Baumeier, F. Huerkamp, T. A. Leskova, and A. A. Maradudin, Phys. Rev. A 84, 013810 (2011).

[23] R. E. Arias and A. A. Maradudin, Opt. Express 21, 9734 (2013).

[24] J. Polanco, R. M. Fitzgerald, and A. A. Maradudin, Plasmonics 10, 1173 (2015).

[25] M. S. Aporvari, A. S. Aporvari, and F. Kheirandish, Appl. Opt. 55, 2375 (2016).

[26] J. Ordonez-Miranda, L. Tranchant, S. Gluchko, T. Antoni, and S. Volz, Phys. Rev. B 90, 155416 (2014).

[27] R. E. Collin, Field Theory of Guided Waves, 2nd ed. (Wiley, New York, 1991). 